Christopher A. Edelmann, Patrick C. Le Clercq, Berthold Noll, Numerical investigation of different modes of internal circulation in spherical drops: Fluid dynamics and mass/heat transfer, International Journal of Multiphase Flow, Volume 95, October 2017, Pages 54-70.

The original publication is available at www.elsevier.com

https://doi.org/10.1016/j.ijmultiphaseflow.2017.05.005

(C) 2017. This manuscript version is made available under the CC-BY-NC-ND 4.0 license http://creativecommons.org/licenses/by-nc-nd/4.0/ 


\title{
Numerical investigation of different modes of internal circulation in spherical drops: Fluid dynamics and mass/heat transfer
}

\author{
Christopher A. Edelmann, Patrick C. Le Clercq, Berthold Noll \\ DLR Stuttgart, Pfaffenwaldring 38-40, 70569 Stuttgart, Germany
}

\begin{abstract}
The results of detailed, three-dimensional numerical simulations of fixed spherical drops in a uniform flow are presented. The fluid dynamics outside and inside of the drops as well as the internal problem of mass (or heat) transfer are studied. Liquid drops in both a liquid and a gaseous ambient phase are considered. Special emphasis is put on the investigation of different modes of internal circulation.

At low Reynolds numbers of the inner fluid, the flow field inside the drop resembles the well known Hill's vortex solution. However, at higher internal Reynolds numbers, stable steady or quasi-steady alternative modes of internal circulation are found. As these modes are not cylindrical symmetric around the streamwise axis, the often applied assumption of a two-dimensional, axisymmetric flow field is not justified in these cases. Thus, major discrepancies to previous numerical studies are obtained. However, it is shown that experimental results support our findings.

For liquid drops surrounded by a liquid, a major influence of the state of internal circulation on the drag is discovered, whereas the drag is nearly unaltered in the case of a liquid drop in gas.
\end{abstract}

Email address: christopher.edelmann@dlr.de (Christopher A. Edelmann) 
Concerning the internal problem of mass/heat transfer, the various internal flow modes show different characteristics. At low internal Peclet numbers, higher Sherwood numbers are reached for the Hill's vortex-like cases, whereas at higher Peclet numbers, the transfer is faster for the alternative modes. For cases with a Hill's vortex-like solution, asymptotic Sherwood numbers for very high Peclet numbers of around 20 are found, whereas no upper limit for cases with alternative modes can be determined. In the present study a maximum internal Sherwood number of 130 is reached, more than six times the maximum value for a case with a Hill's vortex-like internal solution.

Keywords: Drop, Modes of Internal Circulation, Drag Coefficient, Heat/Mass Transfer, CFD

\section{List of symbols}

\section{Latin letters}

$\begin{array}{ll}C_{D} & \text { drag coefficient } \\ C_{L} & \text { lift coefficient } \\ c & \text { concentration } \\ \tilde{c} & \text { volume averaged concentration } \\ d & \text { diameter of the drop (sphere) } \\ D & \text { diffusion coefficient } \\ f & \text { frequency } \\ F_{D} & \text { drag force } \\ F_{L} & \text { lift force } \\ \dot{m}^{\prime} & \text { normalized mass (heat) flux } \\ N u & \text { Nusselt number } \\ p & \text { pressure } \\ P e & \text { Peclet number } \\ P r & \text { Prandtl number }\end{array}$




$\begin{array}{ll}R e & \text { outer Reynolds number } R e=\frac{U_{o} d \rho_{o}}{\mu_{o}} \\ R e_{i} & \text { inner Reynolds number } R e_{i}=\frac{U_{i} d \rho_{i}}{\mu_{i}} \\ S h & \text { Sherwood number } \\ S t & \text { Strouhal number } S t=\frac{f d}{U_{o}} \\ t & \text { time } \\ t^{\prime} & \text { nondimensional time (Fourier number) } t^{\prime}=4 \frac{D t}{d^{2}} \\ U & \text { absolute velocity of the fluid } \\ U_{i} & \text { governing inner velocity } \\ & \text { (maximum absolute velocity inside the drop) } \\ U_{o} & \text { governing outer velocity (inflow velocity) } \\ \mathbf{v} & \text { velocity vector }\end{array}$

\section{Greek letters}

$\begin{array}{ll}\rho & \text { density } \\ \mu & \text { viscosity } \\ \lambda & \text { viscosity ratio inner to outer fluid } \\ \kappa & \text { density ratio inner to outer fluid }\end{array}$

\section{Subscripts}

$\begin{array}{ll}c f & \text { creeping flow } \\ f & \text { friction (viscous) component } \\ i & \text { inside the drop } \\ o & \text { outside the drop } \\ p & \text { pressure (form) component }\end{array}$

\section{Symbols}

- averaged quantity

$\infty \quad$ asymptotic value in the limit $t \rightarrow \infty$ 


\section{Introduction}

Drops have been studied for a long time in various areas and a vast amount of literature on this topic exists. An introduction as well as a review of work until 1978 can be found in the book by Clift et al. (1978). Wegener et al. (2014) present a more recent review covering the fluid dynamics and the mass transfer of drops in liquid-liquid systems. Concerning the present study, the literature can be divided into two parts. First, studies of the internal motion and its influence on drag, and second studies of the internal problem of heat/mass transfer. As the early theoretical and experimental work is reviewed in Clift et al. (1978) and the internal droplet motion is hardly accessible by experiments, mainly numerical studies are cited. However, one experimental investigation should be mentioned, which is the work by Thorsen et al. (1968) who studied the terminal velocity of liquid drops falling through water. They found that for some substances a sudden increase of terminal velocity occurs at a certain drop diameter and attributed this phenomena to "different states or levels of internal circulation".

Early numerical works covering the motion inside drops include the investigation by LeClair et al. (1972) who were the first to numerically study a water drop in air, Abdel-Alim and Hamielec (1975) who studied liquid drops in a liquid, and Rivkind and Ryskin (1976) who considered arbitrary ratios of inner to outer viscosity $(\lambda)$ and outer Reynolds numbers of up to $R e=200$. Oliver and Chung (1987) verified the early numerical results and studied the fluid mechanics in more detail. Feng and Michaelides (2001) used a method to highly resolve the boundary layer outside of the sphere and simulated cases for viscosity ratios between 0 and $\infty$ and $R e<1,000$. In all above mentioned studies, two-dimensional, axisymmetric simulations were performed. It was found that the internal motion is rather insensitive to the internal Reynolds number $R e_{i}$ 
and resembles in many cases a Hill's vortex (Hill (1894)). For certain cases, e.g. a water drop in air at $R e=300$, a secondary circular internal motion emerges in the rear part. Furthermore, Feng and Michaelides (2001) stated that the density ratio $(\kappa)$ of inner to outer density has nearly no influence on drag. Sugioka and Komori (2007) simulated water drops in air with three-dimensional simulations. At $R e=300$, they found that even for a uniform air flow, the outer as well as the inner flow field are not axisymmetric. They showed that the secondary internal motion, described by LeClair et al. (1972), is only possible because they assume axisymmetry and is nonexistent if simulated with a full three-dimensional method. Engberg and Kenig (2015) numerically investigated the observation made by Wegener et al. (2010) that in their experiments, in some cases, two different rise velocities were found for the same drop diameter. Even though their main objective was the deformation of the drops, they found a non-axisymmetric solution for nearly spherical drops and drew the conclusion that "the widely-used assumption of axial symmetry has to be carefully checked".

As we are mainly interested in the phenomena inside the drop, only the internal problem of heat/mass transfer will be discussed. The internal problem is of importance if the main transfer resistance is located inside the drop. The heat/mass transfer inside the drop is characterized using the internal Nusselt (heat transfer) or Sherwood (mass transfer) number, denoted by $N u$ and $S h$, respectively. As these numbers are used analogously, only the Sherwood number is used in the following.

For the case of pure diffusion inside the drop, Newman (1931) derived an analytical solution and obtained an asymptotic value for $t \rightarrow \infty: S h_{\infty}(P e=0) \approx 6.58$, with Pe denoting the Peclet number. Kronig and Brink (1951) assumed creeping flow and very large Peclet numbers inside the drop and thus obtained a solu- 
tion for $R e \rightarrow 0$ and $P e \rightarrow \infty$, which leads to the result $S h_{\infty}(R e \rightarrow 0, P e \rightarrow$ $\infty) \approx 17.9$. This result can be interpreted as the fact that the "overall heat and mass transfer rate between the droplet interior and the surface is 2.72 times higher than in the case of the solid sphere" (Abramzon and Sirignano (1989)). This approach is called the "effective conductivity model", and mainly concerns the finding of the factor $\chi$ by which the sphere thermal conductivity is increased (see Jin and Borman (1985), Talley and Yao (1988)), usually ranging from $\chi=1(S h=6.58)$ to $\chi=2.72(S h=17.9)$. However, only creeping flow is assumed in the aforementioned studies. Handlos and Baron (1957) assumed that the streamlines inside the drop can be approximated using the Hill's vortex solution but superimposed turbulent fluctuations, as they considered very high Reynolds numbers. As a result, they obtained a linear relationship of the Sherwood number as a function of the Peclet number. More recently, Paschedag et al. (2005) studied the influence of changes in material properties and operating conditions on the mass transfer and found only small influence of the Reynolds number on mass transfer. Juncu (2010) studied the influence of very high Peclet and moderately high Reynolds numbers on the transfer and stated that $S h_{\infty}\left(R e_{i}, P e \rightarrow \infty\right)$ increases with increasing inner Reynolds number. Their highest result obtained was for a liquid drop with $\lambda=100, \kappa=1,000$, yielding $S h_{\infty}\left(R e_{i}=1,000, P e=100,000\right)=19.85^{1}$, and exceeding the results from Kronig and Brink (1951) by only approximately 10\%. Colombet et al. (2013) studied spherical gas bubbles at moderate Reynolds numbers. They found that the maximum tangential velocity at the bubble surface is the governing parameter for the description of the transfer and were able to show that all results collapse on a single curve if a appropriately normalized asymptotic Sherwood number is plotted as a function of maximum Peclet number $P e_{\max }$.

\footnotetext{
${ }^{1}$ Note that they use a different definition for $R e_{i}$ with $R e_{i}=R e \cdot \kappa / \lambda$.
} 
For the present study, three-dimensional numerical simulations of fixed spherical drops were performed with the well validated finite-volume code THETA (see e.g. Knopp et al. (2010)). The main purpose of the this paper is to show that for physical relevant parameters, solutions inside spherical liquid drops exist, which do not resemble a Hill's vortex. In particular, it presents in which cases this may occur and how the drag and the internal heat/mass transfer are influenced. The paper is structured as follows. Section 2 covers the used model and the governing equations, whereas section 3 describes the numerical method. In section 4, the basic validation and the mesh quality are discussed, followed by the main part (section 5), covering the results and the discussion. The conclusions can be found in section 6 .

\section{Model and Governing Equations}

We consider a fixed spherical drop with diameter $d$, placed in a uniform flow with inflow velocity $U_{o}$. The flow fields inside and outside the drop are governed by the unsteady incompressible Navier-Stokes equations for non-reacting Newtonian fluids with constant viscosity and density, without external forces. They can be written in vector notation as:

$$
\begin{aligned}
0 & =\nabla \cdot \mathbf{v} \\
\rho \frac{D \mathbf{v}}{D t} & =\mu \nabla^{2} \mathbf{v}-\nabla p
\end{aligned}
$$

where $\mathbf{v}$ is the velocity vector, $\rho$ is the density, $\mu$ is the viscosity and $p$ is the pressure. The flow fields inside and outside of the drop are separated by the

drop surface, where a discrete jump in viscosity and density occurs. The ratios 
between the inner and the outer fluids are:

$$
\begin{aligned}
\lambda & =\frac{\mu_{i}}{\mu_{o}} \\
\kappa & =\frac{\rho_{i}}{\rho_{o}}
\end{aligned}
$$

where the subscripts $o$ and $i$ denote the outer and the inner fluids, respectively. Besides for validation purposes, $\lambda$ and $\kappa$ are either chosen to be both in the order of one (liquid drop surrounded by a liquid) or $\lambda \geq 20$ and $\kappa \gg 100$ (liquid drop surrounded by a gas). At the fluid-fluid interface, the total velocity and the shear stress are continuous. As the position of the interface is fixed and there is no interfacial mass transfer, the normal velocities of both phases at the interface are zero. Furthermore, both the tangential velocities and the shear stresses match:

$$
\begin{gathered}
v_{n, o}=v_{n, i}=0 \\
v_{t, o}=v_{t, i} \\
\mu_{o} \frac{\partial v_{t, o}}{\partial n}=\mu_{i} \frac{\partial v_{t, i}}{\partial n}
\end{gathered}
$$

where $n$ is the coordinate normal to the interface and the subscripts $n$ and $t$ denote the normal and the tangential components, respectively.

The outer and inner Reynolds numbers are defined as

$$
\begin{gathered}
R e=R e_{o}=\frac{U_{o} d \rho_{o}}{\mu_{o}} \\
R e_{i}=\frac{U_{i} d \rho_{i}}{\mu_{i}}
\end{gathered}
$$

where $U_{i}$ is the maximum absolute velocity inside the drop $\left(\left|\mathbf{v}_{i}\right|_{\max }\right)$, which is for all cases equal to the maximum absolute velocity at the drop surface. For the present study it is defined that $d \cdot \rho_{0} / \mu_{0}=1$, which means that $R e=R e_{0}=U_{0}$. As the drop is fixed and the inflow is uniform, the drag and lift coefficients each consist of two parts. A pressure (or form) part (denoted by the subscript $p$ ) and 
a friction (or viscous) component (denoted by the subscript $f$ ):

$$
\begin{gathered}
C_{D}=C_{D, p}+C_{D, f}=8 \frac{F_{D, p}+F_{D, f}}{\pi \rho_{o} U_{o}^{2} d^{2}} \\
C_{L}=C_{L, p}+C_{L, f}=8 \frac{F_{L, p}+F_{L, f}}{\pi \rho_{o} U_{o}^{2} d^{2}}
\end{gathered}
$$

where $C_{D}$ and $C_{L}$ are the drag and the lift coefficients, respectively and $F_{D}$ and $F_{L}$ are the drag and the lift forces, respectively.

For the transport of the mass/heat inside the drop, the independent nondimensional scalar $c$ (for concentration) is used. It spans from zero, which is the initial condition inside the drop, up to one, which is the fixed condition at the drop surface. It is assumed that a change in concentration does not change any fluid properties, and thus the equation for the transport of $c$ is decoupled from the Navier-Stokes equations. The transport of the concentration inside the drop is governed by:

$$
\frac{\partial c}{\partial t}+\mathbf{v} \cdot \nabla c=D \nabla^{2} c
$$

where $D$ is the diffusion coefficient. The governing time-scale for the transport process is the Fourier number (nondimensional time):

$$
t^{\prime}=4 \frac{D t}{d^{2}}
$$

The average Sherwood (or Nusselt) number inside the drop is given as a function of the Fourier time by:

$$
\operatorname{Sh}\left(t^{\prime}\right)=\frac{2}{3} \frac{1}{1-\tilde{c}} \frac{d \tilde{c}}{d t^{\prime}}
$$

(Clift et al. (1978)), where $\tilde{c}$ is the volume averaged concentration. For the Peclet number, two definitions are used:

$$
\begin{gathered}
P e=\frac{U_{o} d}{D} \\
P e_{i}=\frac{U_{i} d}{D}
\end{gathered}
$$


In our opinion, the definition for $P e_{i}$ (Equation (16)) is in many cases preferable as the time scale of the transport by convection inside the drop is governed by the velocities inside the drop. However, Pe from Equation (15) has the advantage of being known in advance, whereas for $P e_{i}$ the maximum absolute velocity at the drop surface in the general case is not known a priori. In the creeping flow limit, the maximum velocity inside the drop is given by $U_{i}=U_{o} /(2(1+\lambda))$ and thus $P e_{i, c f}=P e /(2(1+\lambda))$.

\section{Numerical method}

The three-dimensional simulations of the flow inside and outside the drop, as well as the transport of the concentration were performed with the German Aerospace Center (DLR) inhouse code THETA. THETA possesses compressible and incompressible finite-volume solvers for steady and unsteady problems and works on unstructured grids. It is well validated and used for a variety of computational fluid dynamic (CFD) problems. Examples are Unsteady ReynoldsAveraged Navier-Stokes (URANS) simulations of multiphase flows (Boyde et al. (2013), Eckel et al. (2016)) or Scale Adaptive Simulations (SAS) of mixing in jet in crossflow configurations (Ivanova et al. (2009)) or Large Eddy Simulations (LES) of the flow over a backward facing step (Knopp et al. (2010)). Furthermore, it allows for the transport of arbitrary species, handles strongly varying density fields and is routinely used for combustion simulations (e.g. Domenico et al. (2010)). Recently a $4^{\text {th }}$-order as well as a $2^{\text {nd }}$-order low dissipation scheme for the spatial discretization were implemented by Löwe et al. (2015).

For the present simulations, the convective as well as the diffusive fluxes were discretized using central schemes. For the velocity in the momentum fluxes the $4^{\text {th }}$-order low-dissipation low-dispersion scheme by Löwe et al. (2015) was used. The Crank-Nicolson scheme was selected for the time-discretization. For the splitting of the pressure and the velocity calculations, the incremental variant 
of the projection method is applied. Convergence in time was carefully checked by monitoring the residuals of the pressure and velocity as well as the drag force. The drop is situated in the middle of a three-dimensional, cubic computational domain. At the inflow boundary plane a uniform velocity $U_{o}$ is prescribed, whereas at the outflow a condition is used where the pressure is fixed and the velocities are extrapolated. At the side planes, symmetry conditions are applied. The simulations are run until a steady or quasi-steady solution is found. Only then, the simulations of the transport of the mass/heat inside the drop are started by prescribing the fixed condition for concentration $(c=1)$ at the drop surface (see section 2). The simulations are continued until the average concentration inside the drop reaches a clear asymptotic Sherwood number value or $\tilde{c}=0.999$.

A section of a typical grid used for the present simulations is shown in Figure 1. The mesh is highly refined near the drop surface, with the same height for the first elements $(\Delta)$ on both sides of the interface. In Table 1, the most important parameters for the meshes used in the present study can be found. Mesh 1 is the reference mesh and the other two are derived from that mesh. Mesh 2 is refined both outside and inside, whereas Mesh 3 is unchanged outside the sphere but highly refined inside. The first grid spacing from the fluid-fluid interface was choosen such that the velocity as well as the concentration boundary layer are sufficiently resolved. The boundary layer thicknesses were estimated by $\delta \sim R e^{-1 / 2} d$ for the velocity boundary layer and by $\delta \sim P e^{-1 / 2} d$ for the concentration boundary layer. Even for Reynolds or Peclet numbers up to 10,000, $\Delta / d$ in all three meshes (see Table 1 ) is well below the estimated boundary layer thickness of about 0.01 . For all meshes, the domain size is $40 d$ in all three spatial dimensions. The size was chosen based on a literature review (see e.g. Johnson and Patel (1999) or Sugioka and Komori (2007)) and validated to be 


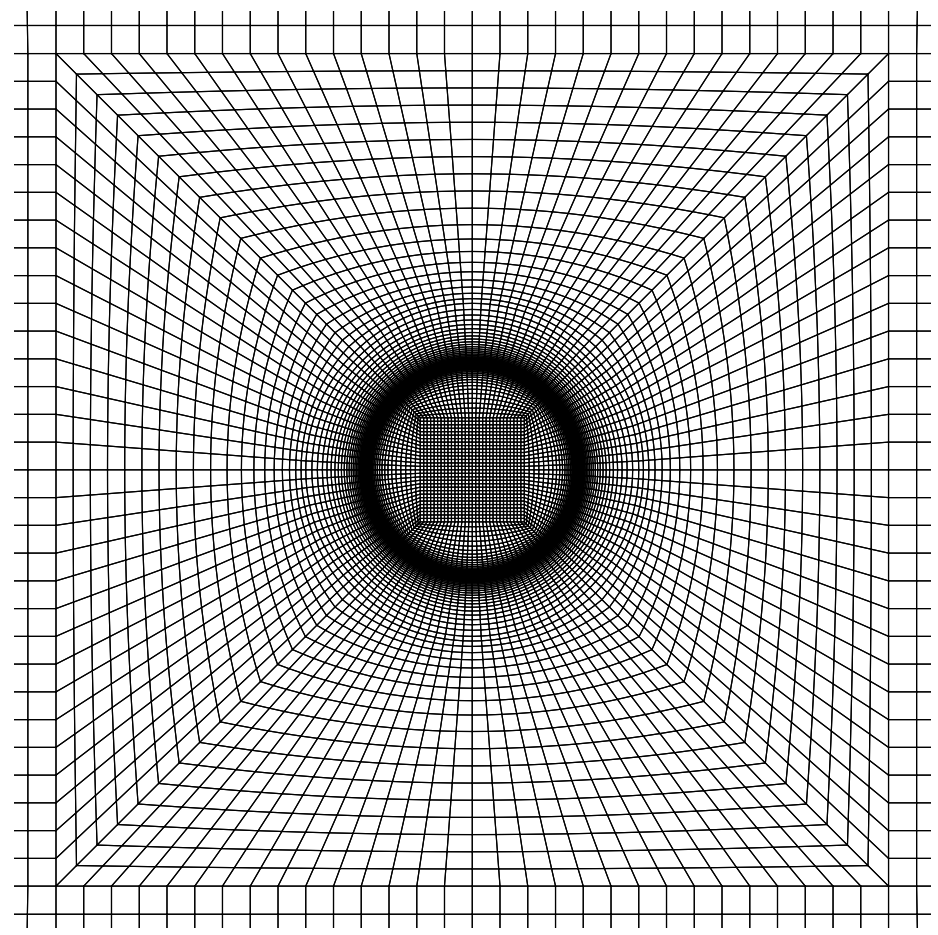

Figure 1: Grid (Mesh 1) in vicinity of the drop. A section of a $x-y$ slice through the origin is shown. 
Table 1: Mesh characteristics.

$N_{\text {in }}$ and $N_{\text {out }}$ are the number of elements inside and outside of the fluid sphere, respectively. $\Delta$ is the grid spacing of the first element at the sphere surface (inside and outside). L denotes the length, $\mathrm{H}$ the height and $\mathrm{W}$ the width of the computational domain.

\begin{tabular}{lcccl}
\hline & $N_{\text {in }}$ & $N_{\text {out }}$ & $\frac{\Delta}{d}$ & $\frac{L}{d} \times \frac{H}{d} \times \frac{W}{d}$ \\
\hline Mesh 1 & 129,600 & $1,637,128$ & 0.002 & $40 \times 40 \times 40$ \\
Mesh 2 & 149,850 & $2,501,775$ & 0.001 & $40 \times 40 \times 40$ \\
Mesh 3 & 205,200 & $1,637,128$ & 0.002 & $40 \times 40 \times 40$ \\
\hline
\end{tabular}

sufficient for $R e \geq 5$ (see section 4$)$.

\section{Basic Validation and Mesh Convergence}

The DLR code THETA is well validated and in use for a variety of CFD problems (see section 3). However, it was never before used for the detailed simulation of the conjugate flows inside and outside of drops. Thus, before the discussion of the main results, a basic validation of the code for this purpose is necessary. In the following, we present a comparison with published results, as well as a grid study. The fluid dynamics on the one hand and the internal problem of mass/heat transfer on the other hand are discussed separately.

\subsection{Fluid Dynamics}

First of all, the mesh quality is checked. The drag coefficient for viscous and solid spheres for some combinations of $R e, \lambda$ and $\kappa$ are shown in Table 2 . The cases were simulated on two different meshes, with Mesh 2 being the refined mesh (see section 3 for more details). It can be seen that the relative difference in drag between cases simulated on both meshes increase with increasing Reynolds number, but that it stays well below 1\%, even for the highest Reynolds number calculated. 
Table 2: Drag coefficient for viscous and solid spheres for combinations of $R e, \lambda$ and $\kappa$, simulated on Mesh 1 and Mesh 2. $\lambda=\infty$ indicates a solid sphere.

\begin{tabular}{cccccc}
\hline$R e$ & $\lambda$ & $\kappa$ & Mesh 1 & Mesh 2 & Deviation in \% \\
\hline 100 & $\infty$ & - & 1.090 & 1.089 & 0.04 \\
300 & $\infty$ & - & 0.657 & 0.658 & 0.18 \\
138 & 0.8 & 1.107 & 0.480 & 0.480 & 0.07 \\
318 & 0.8 & 1.107 & 0.382 & 0.380 & 0.43 \\
632 & 0.8 & 1.107 & 0.354 & 0.352 & 0.76 \\
100 & 20 & 20 & 1.054 & 1.053 & 0.09 \\
300 & 49 & 829 & 0.632 & 0.631 & 0.24 \\
\hline
\end{tabular}

Another very basic problem is the definition of the size of the computational domain. For this purpose, Mesh 1 was extended in all three dimensions such that the domain size is $80 d$ in each direction. A case with a viscous sphere at $R e=5$ was simulated on the original as well as the extended mesh and the difference in drag is below $0.1 \%$.

The flow around solid spheres in a uniform flow is a very well investigated topic. It is known that at around $R e=210$ the axisymmetry is broken down by a regular bifurcation and a steady, plane-symmetric solution emerges, which is stable until approximately $R e=270$. Above $R e=270$ this solution becomes unstable and periodic shedding of vortices occurs (see for example Ghidersa and Dusek (2000) or Bagchi and Balachandar (2002) for more details). As natural non-axisymmetric solutions and the beginning of unsteadiness are important phenomena in the present work, the study of the flow around rigid spheres is selected as a validation scenario.

Simulations of solid spheres at $R e=10,100,200,212,270,280,300$ were conducted. In accordance with the literature, at $R e=10,100,200$ nearly perfectly symmetric solutions are found, whereas at $R e=212$ a slight non- 
Table 3: Drag and lift coefficients, as well as the Strouhal number for solid spheres. The tilde denotes averaged quantities.

\begin{tabular}{cccccc}
\hline & $R e$ & Present & Literature 1 & Literature 2 & Literature 3 \\
\hline$C_{D}$ & 100 & 1.089 & 1.09 & 1.087 & - \\
$\tilde{C}_{D}$ & 300 & 0.658 & 0.650 & 0.656 & 0.657 \\
$\tilde{C}_{L}$ & 300 & 0.064 & - & 0.069 & 0.067 \\
$S t$ & 300 & 0.135 & - & 0.137 & 0.134 \\
\hline
\end{tabular}

Literature 1: Feng and Michaelides (2001). Note: two-dimensional, axisymmetric simulations.

Literature 2: Johnson and Patel (1999).

Literature 3: Kim and Choi (2002).

axisymmetry (detected by the appearance of lift) is observed. At $R e=270$ this phenomenon is quite pronounced but still stable. At $R e=280$ and $R e=300$ an unsteady solution with periodic vortex shedding emerges. In Table 3, drag $\left(C_{D}\right)$ and lift $\left(C_{L}\right)$ coefficients as well as the Strouhal number $(S t)$ of cases at $R e=100$ and $R e=300$ are compared with results from the literature. Note that the tilde denotes averaged quantities. A good agreement with numerical results from the literature is found. Simulations of fluid spheres are compared with results from Feng and Michaelides (2001) for some combinations of Re, $\lambda$ and $\kappa$ listed in Table 4. Feng and Michaelides (2001) were chosen as they validated their results against both results from numerical simulations and experiments. It can be seen from Table 4 that an excellent agreement is achieved for the cases at $R e=20$ and $R e=100$, whereas the agreement for the case at $R e=200$ is still acceptable. The reason for the relatively large deviation for the last-mentioned case is unknown.

As mentioned above, only very few data exists of non-axisymmetric solutions for fluid spheres. Sugioka and Komori (2007) show that the flow field both inside and outside of a spherical water drop in a uniform air stream at $R e=300$ does 
Table 4: Comparison of drag coefficients for fluid spheres for some combinations of $R e, \lambda$ and $\kappa$.

\begin{tabular}{cccccc}
\hline$R e$ & $\lambda$ & $\kappa$ & Present & Feng and Michaelides (2001) & Deviation in \% \\
\hline 20 & 1 & 1 & 2.037 & 2.037 & 0 \\
100 & 3 & 3 & 0.873 & 0.878 & 0.58 \\
100 & 20 & 20 & 1.055 & 1.059 & 0.44 \\
200 & 1 & 1 & 0.387 & 0.397 & 2.41 \\
\hline
\end{tabular}

(a)

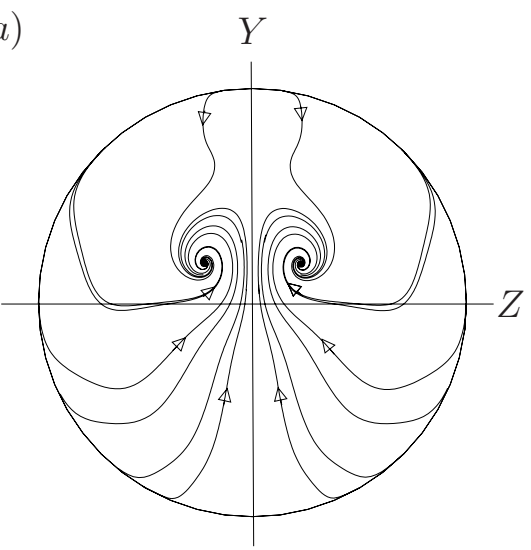

(b)

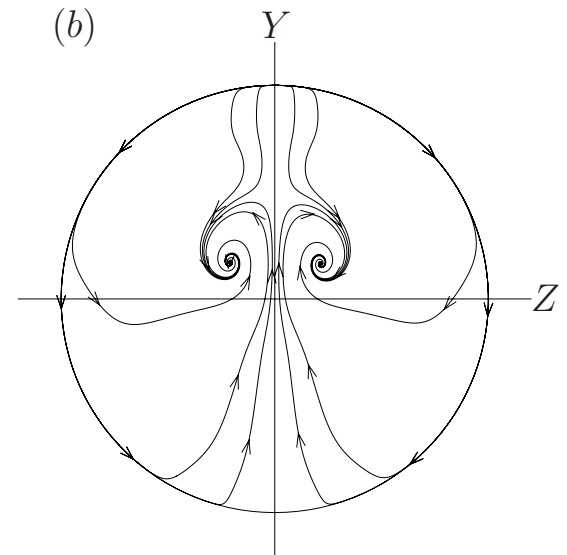

Figure 2: Streamlines in the $y$ - $z$ plane (plane perpendicular to the inflow vector) through the origin inside a fluid sphere with $\lambda=49, \kappa=829$ at $R e=300$ are shown.

(a) Simulation by Sugioka and Komori (2007).

(b) Present simulation.

not exhibit an axial symmetry, but it contains a plane of symmetry. In Figure 2 their solution is compared with a simulation from the present work. The exact placement of the streamlines is different, but it can be clearly seen that the flow features are very similar.

\subsection{Internal Problem of Mass/Heat Transfer}

The very basic problem of heat or mass transfer inside a drop is that of the pure diffusion problem, meaning that $P e=0$. In Figure 3, present simulation results for the concentration versus radial position at different times are compared with the analytical solution of Newman (1931). A nearly perfect match 
is achieved. Concerning the Sherwood number in the limit $t \rightarrow \infty\left(S h_{\infty}\right)$, the analytical solution gives $S h_{\infty}=6.58$, whereas the asymptotic result from our simulation is $S h_{\infty}=6.57$.

Even though liquid drops are researched, the present method works with bubbles as well. A quite recent study by Colombet et al. (2013) concerns the transfer inside a spherical gas bubble. They validated their results and presented appropriate data to compare with. In accordance with Colombet et al. (2013), bubbles with $\lambda=0.018$ and $\kappa=0.0012$ are simulated. In Table 5 , results for $S h_{\infty}$ from Colombet et al. (2013) are compared with results from the present work, simulated on different meshes. Even for the highest Peclet number $(P e=2,000)$ the deviations between the results simulated on different meshes are below $1.5 \%$. Results from the present work are generally higher than the results from Colombet et al. (2013). However, the maximum deviation does not exceed 3.5\% . For further validation, the instantaneous Sherwood number versus nondimensional time $t^{\prime}$ is plotted together with data from Colombet et al. (2013) in Figure 4 for bubbles in the creeping flow regime $(R e=0.1)$. The overall agreement is good and especially the time oscillations due to the convection processes inside the bubble are correctly reproduced. However, distinct differences between both studies can be seen in the parts where the Sherwood number is increasing.

It can be concluded that a good agreement with results from the literature is achieved for both the fluid dynamics and the heat/mass transfer part. Mesh 1 seems sufficiently refined and the domain size adequate. Thus, Mesh 1 is used in the following, if not stated otherwise.

\section{Results and Discussion}

In the results section, we first study the flow structures (section 5.1) and the drag (section 5.2) of liquid drops in a liquid medium, followed by the flow structures (section 5.3) and the drag (section 5.4) of liquid drops in a gaseous 


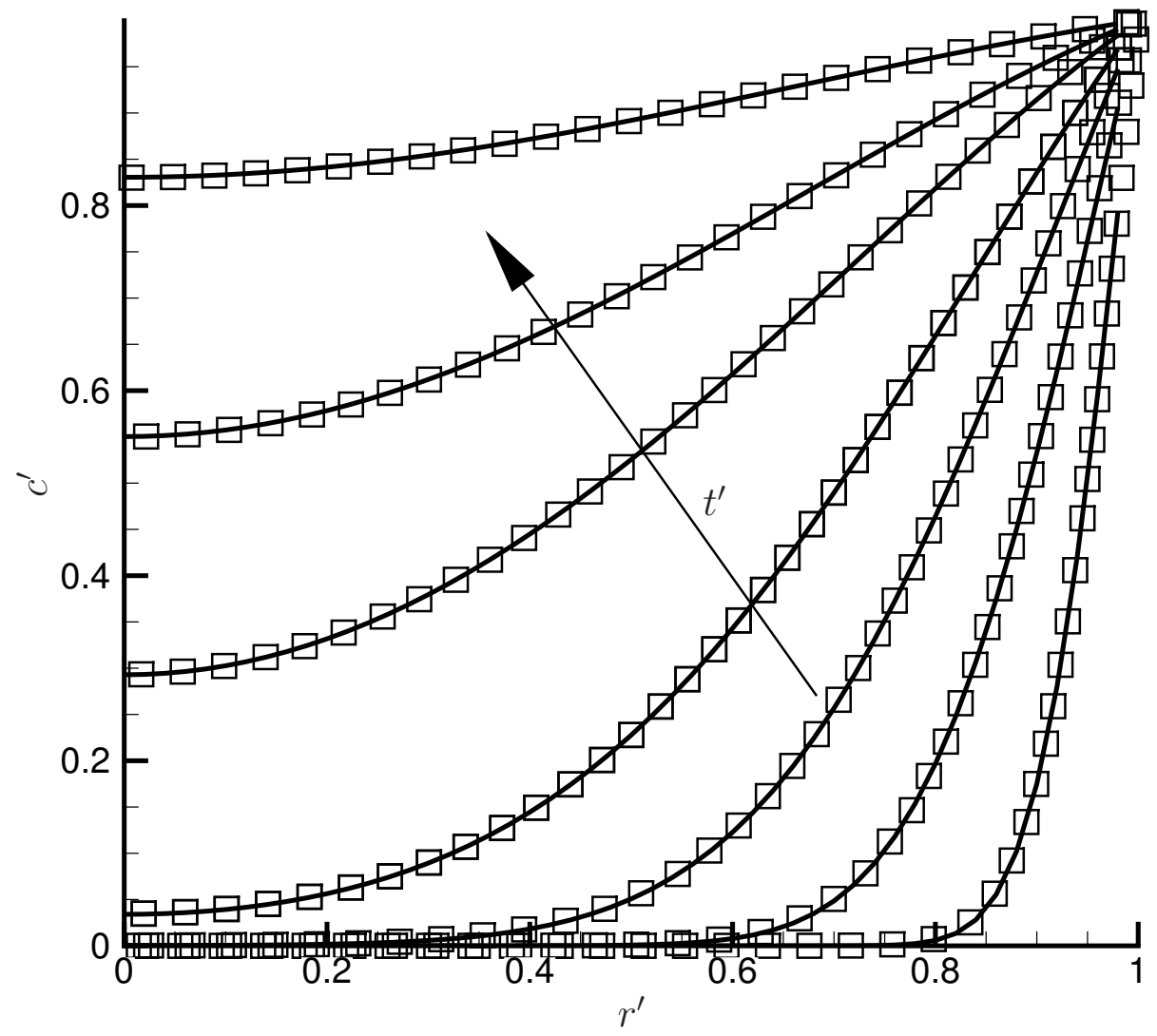

Figure 3: Nondimensional concentration $c^{\prime}$ versus nondimensional radial position $r^{\prime}$ for nondimensional times $t^{\prime}=0.0025,0.01,0.025,0.05,0.1,0.15,0.25$ for the pure diffusion regime. Symbols: present work. Lines: analytical solution of Newman (1931).

Table 5: Sherwood number for $t \rightarrow \infty$ for a bubble, simulated on different meshes and compared with literature: Colombet et al. (2013).

\begin{tabular}{cccccccc}
\hline$R e$ & $\lambda$ & $\kappa$ & $P e$ & Mesh 1 & Mesh 2 & Mesh 3 & Literature \\
\hline 100 & 0.018 & 0.0012 & 20 & 10.46 & 10.46 & 10.46 & 10.31 \\
100 & 0.018 & 0.0012 & 100 & 17.3 & 17.29 & 17.29 & 16.95 \\
100 & 0.018 & 0.0012 & 1,000 & 18.47 & 18.33 & 18.4 & 18.03 \\
100 & 0.018 & 0.0012 & 2,000 & 18.69 & 18.41 & 18.55 & 18.06 \\
\hline
\end{tabular}




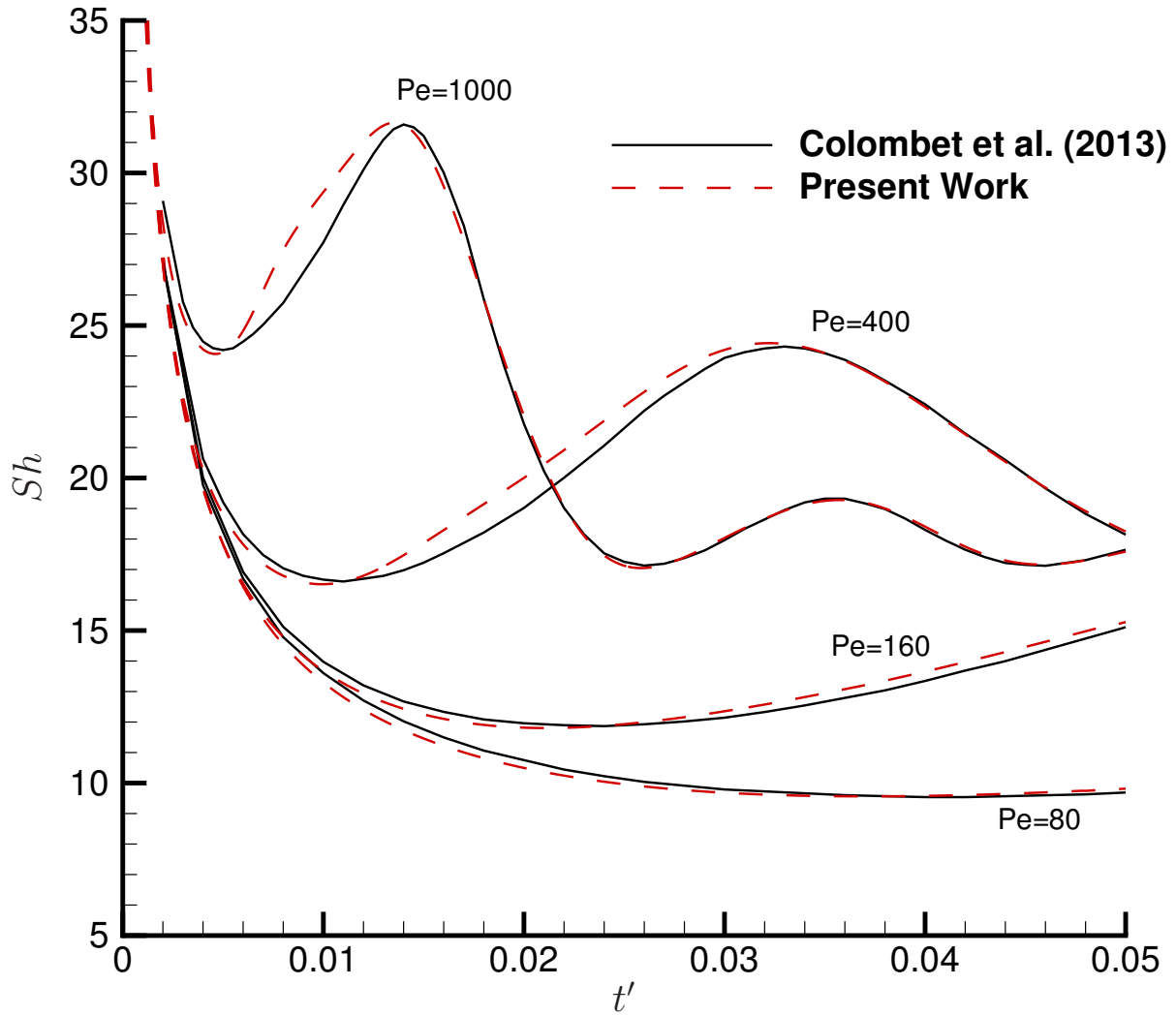

Figure 4: Instantaneous Sherwood number versus nondimensional time $t^{\prime}$ for a bubble at $R e=0.1$. 
medium. At the end of the section, we discuss the internal problem of heat/mass transfer for both systems together (section 5.5).

\subsection{Flow Structures in Liquid-Liquid Systems}

We begin our discussion with liquid-liquid systems. As we are mainly interested in cases where the inner flow structure deviates as much as possible from the Hill's vortex solution, cases are searched with high inner Reynolds number. $R e_{i}$ can be approximated by $R e_{i} \approx R e \cdot \kappa / \lambda$ and thus $\kappa$ should be as big and $\lambda$ as small as possible.

One liquid-liquid system studied by Thorsen et al. (1968) is an ethyl bromide drop in water, which gives at $25^{\circ} \mathrm{C}$ and 1 bar: $\lambda=0.53$ and $\kappa=1.45$. For our present study, we approximate this to $\lambda=0.5$ and $\kappa=1.5$. From the literature (see e.g. Waheed (2001) pages $71-72$ for cases with $\lambda=1$ and $\kappa=1$ ), we can assume that for these parameters, up to at least $R e=200$, no flow separation occurs and the outer flow stays attached to the drop. In Figure 5, streamlines inside and outside the drop in three planes through the origin, as well as the velocities on the drop surface are shown for $R e=100, \lambda=0.5, \kappa=1.5$. It can be seen that the solution is cylindrical symmetric around the $x$-axis. No recirculation zone in the outer flow is visible and the inner flow qualitatively resembles a Hill's vortex. In the outer flow, two stagnation points, one in the front $(S 1)$ and one in the aft $(S 2)$ of the drop are found.

To study the influence of an increasing inner Reynolds number, the outer Reynolds number is increased to 150 , whereas $\kappa$ and $\lambda$ remain constant. According to the literature (e.g. Waheed (2001) or Feng and Michaelides (2001)), the flow field should be similar to the aforementioned case at $R e=100$. However, as shown in Figure 6, a very different solution is obtained. The flow fields both inside and outside the drop are no longer cylindrical symmetric, but now a plane of 
(a)

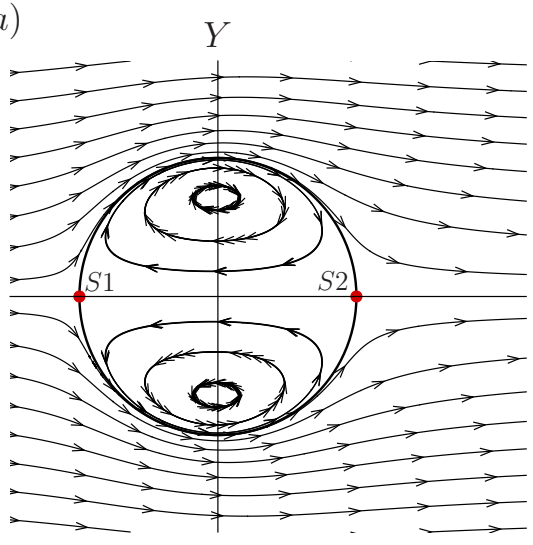

(c)

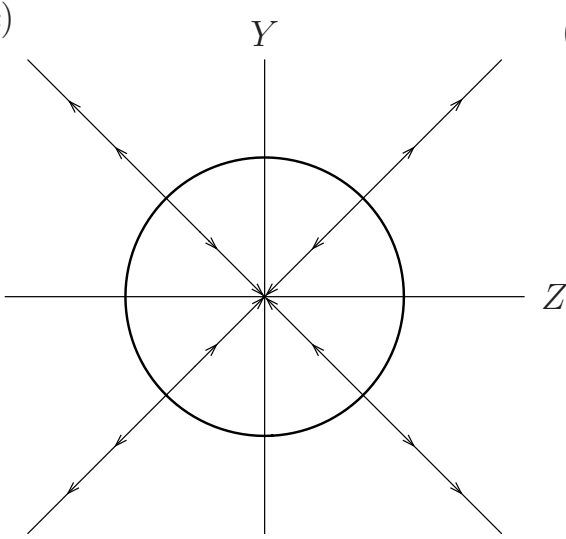

(b)

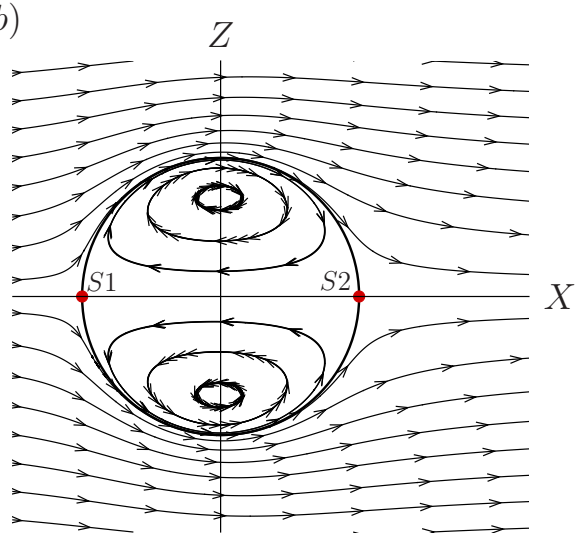

$(d)$

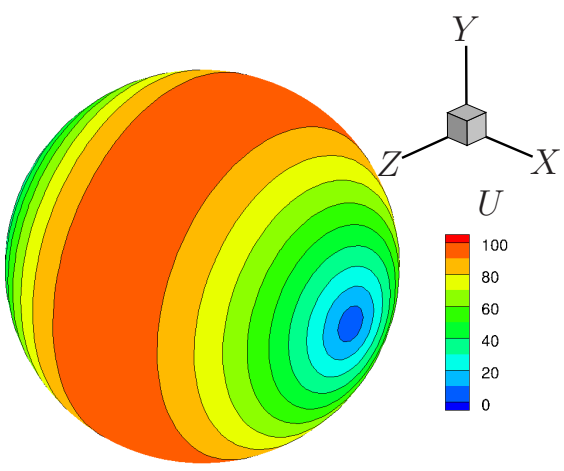

Figure 5: Streamlines (a - c) inside and outside of a fluid sphere as well as contours of absolute velocity at the sphere surface (d) for $R e=100, \lambda=0.5, \kappa=1.5$. In a - c planes through the origin are shown. Red dots indicate stagnation points in the outer flow, denoted by $S 1$ and $S 2$. 
symmetry (the $x-y$ plane) is observed. ${ }^{2}$ Note that in the present case, as well as in the other cases with symmetry plane(s), the plane(s) of symmetry coincide with either the $x-y$ or the $x-z$ plane, whereas in nature the symmetry plane(s) are expected to be arbitrary chosen. We think this is enforced by the grid, the initial conditions without artificial disturbances, and the boundary conditions of our box-shaped computational domain. Similar observations are reported by e.g. Johnson and Patel (1999). For the inner flow, two vortical structures can be seen in the $x-y$ plane in the regions where the flow directions change from clockwise to counterclockwise in the upper half and vice versa in the lower half. In contrast to the cylindrical symmetric case (see Figure $5 \mathrm{c}$ ), flows in the angular direction are observed in the $y$ - $z$ plane (Figure $6 \mathrm{c}$ ), caused by four pairwise counterrotating vortical structures. Another important observation is difficult to show with plots. It seems that all fluid particles inside one of the two hemispheres, divided by the symmetry plane (the $x-y$ plane), are connected by a single three-dimensional streamline. This might influence the heat/mass transfer inside the drop and is a major difference to Hill's vortex-like cases, where a fluid particle stays on the same closed two-dimensional streamline. The outer flow field in the $x-z$ plane (Figure $6 \mathrm{~b}$ ), looks similar to the case at $R e=100$ (Figure $5 \mathrm{~b}$ ), whereas a widening of the streamlines is observed in the $x-y$ plane (Figure 6 a), due to a change in flow direction on the sphere surface. This means that the flow separates and at the separation line, two additional stagnation points, denoted by $S 3$ and $S 4$ are found. No typical trailing vortex is formed, but due to the four vortices inside the drop in the $y$ - $z$ plane (Figure 6 c), four vortices in the wake of the sphere originate. This flow feature is not explicitly visualized in the Figure 6 nor in the following figures but it directly

\footnotetext{
${ }^{2}$ The $y$ - and $z$-axis in the plots in Figure 6 are permuted to compare more easily with following plots, as we assume that the axis orientation is arbitrary.
} 
follows from the illustrations shown.

Keeping $\kappa$ and $\lambda$ constant, the Reynolds number is further increased to 200. As can be seen from Figure 7, the features of the flow field changed again compared to the case at $R e=150$ (see Figure 6 ). Now, two planes of symmetry are found, both the $x-y$ and the $x-z$ plane. (Which means the flow field is also two-fold rotational symmetric around the $x$-axis.) The flow widening and thus the separation in the $x-y$ plane is more pronounced, as the upper stagnation point (S4) moved upwards. It can be seen in Figure 7 a) that the vortical structure in the upper part (positive $y$-coordinate) shrunk compared to the aforementioned case (see Figure 6 a). As the vortical structure in the lower part remained nearly unaltered it now displays a plane symmetry. A similar observation can be made in Figure $7 \mathrm{c}$ ) in the $y$ - $z$ plane. Compared to the case at $R e=150$, the structures in the lower part are similar, whereas in the upper part (positive $y$-coordinate), the structures grew and are now plane symmetric to the structures in the lower part (negative $y$-coordinate). The rotation direction in the $y$ - $z$ plane implies that fluid is transported away from the $x-y$ plane to the $x$ - $z$ plane. According to mass continuity, this means higher velocities in the $x$ - $z$ plane, a fact visualized in Figure $7 \mathrm{~d}$ ). Similar to the case at $R e=150$, each fluid particle inside one of the four quarter-spheres seems to be connected by a single streamline.

If the Reynolds number is further increased, at $R e=250$ a steady state solution with two symmetry planes is found. At $R e=300$ the flow field is minimally unsteady. As the solution is quasi-steady, a snapshot is used for the visualization of the flow field in Figure 8. It can be seen that the flow field is still very similar to the flow field at $R e=200$ (see Figure 7). Besides the minimal unsteadiness, the plane-symmetry is lost. However, the two-fold rotational symmetry around the $x$-axis is retained. Note that the stagnation points $S 3$ and $S 4$ are not shown in the $x-y$ plane (Figure 8 a) as they lie slightly out of the plane. 
(a)

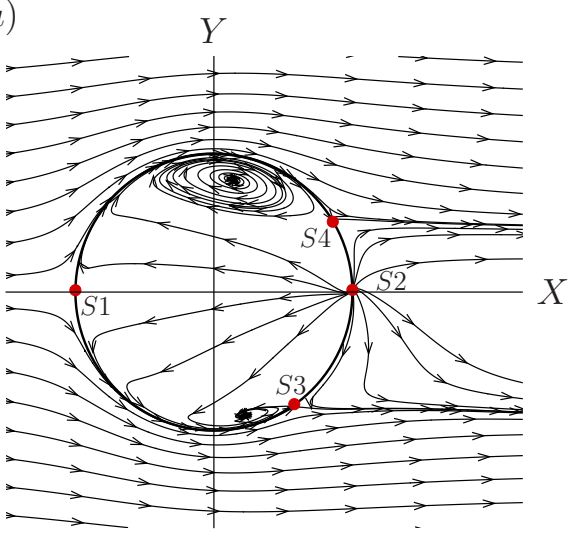

(c)

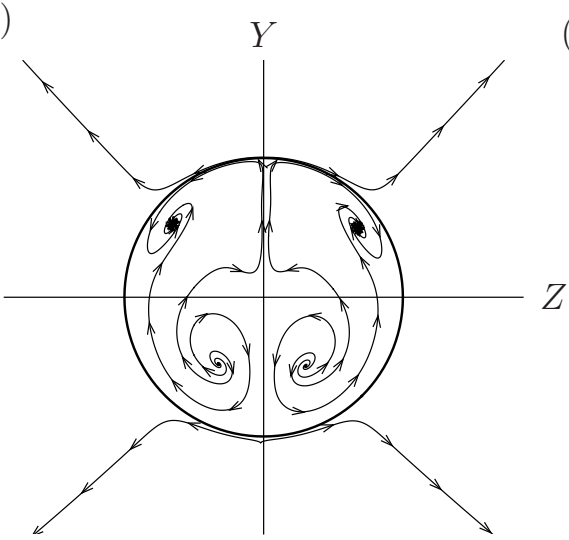

(b)

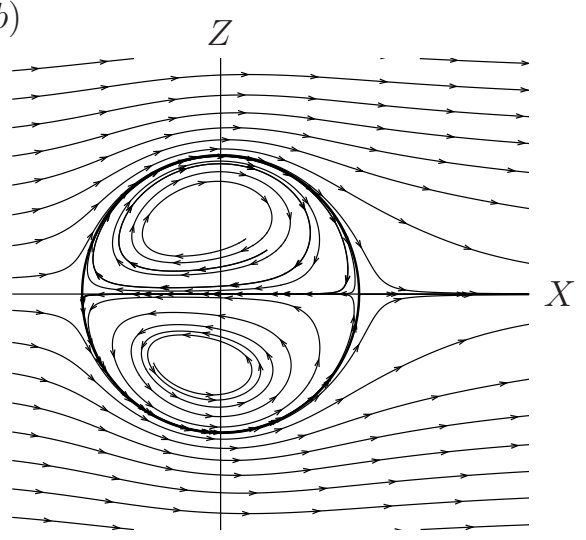

(d)

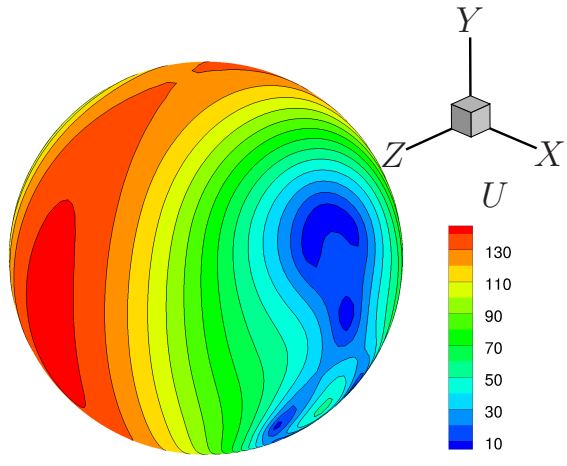

Figure 6: Streamlines (a - c) inside and outside of a fluid sphere as well as contours of absolute velocity (d) at the sphere surface for $R e=150, \lambda=0.5, \kappa=1.5$. In a - c, planes through the origin are shown. Stagnation points in the outer flow are indicated by red dots and denoted by $S 1, S 2, S 3, S 4$. In (b) no stagnation points are visible as they are slightly off the plane. The $y$ - and $z$-axis are permuted to allow easier comparison with following plots. 
(a)

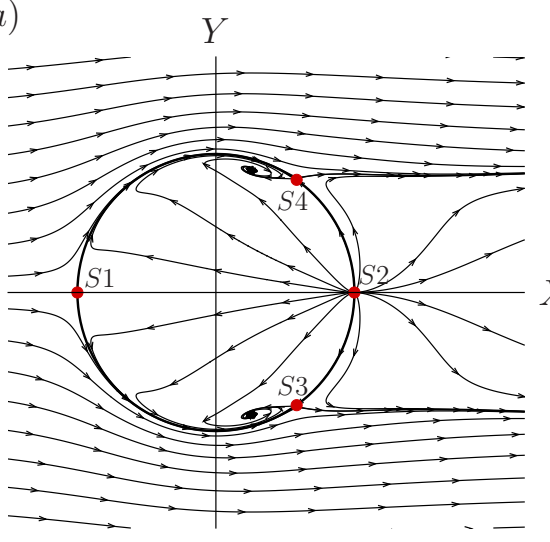

(c)

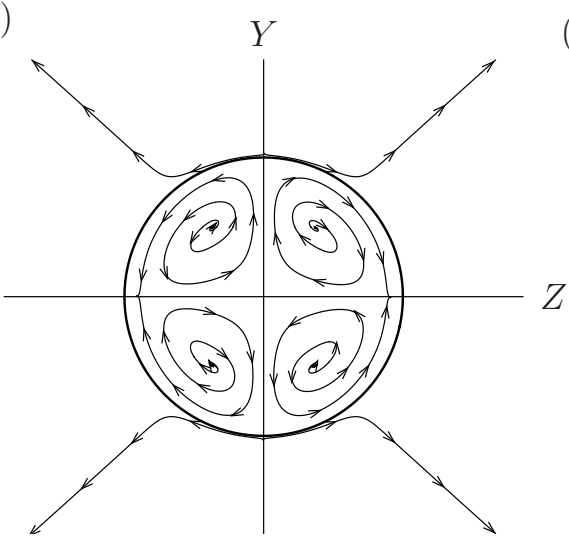

(b)

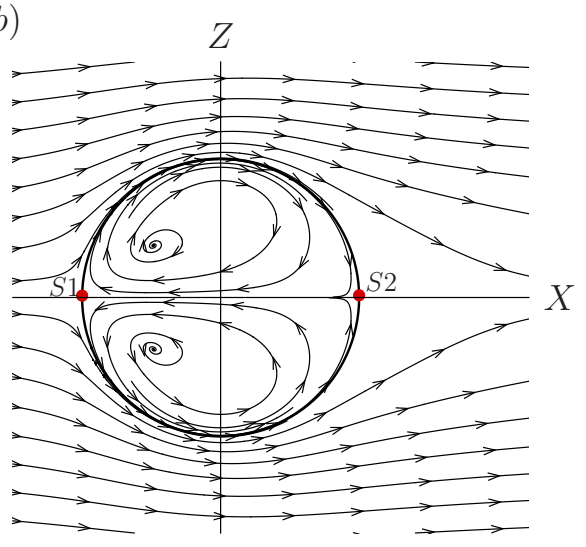

$(d)$

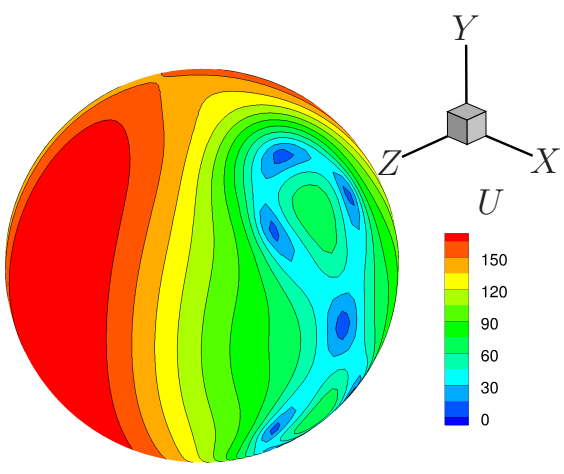

Figure 7: Streamlines (a - c) inside and outside of a fluid sphere as well as contours of absolute velocity (d)) at the sphere surface for $R e=200, \lambda=0.5, \kappa=1.5$. In a - c, planes through the origin are shown. Red dots indicate stagnation points in the outer flow, denoted by $S 1$, $S 2, S 3, S 4$ 
(a)

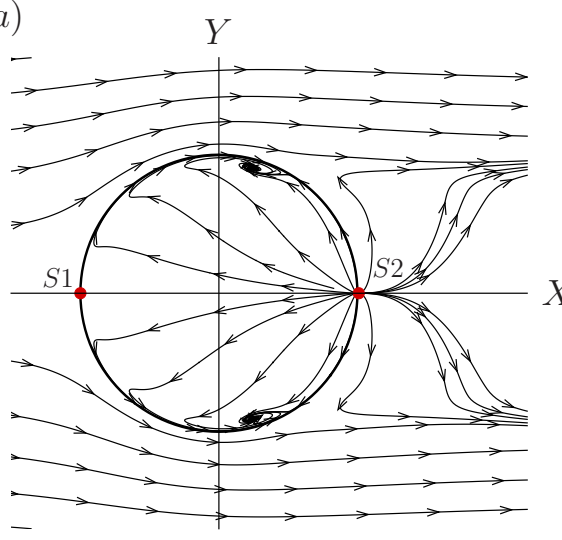

$(c)$

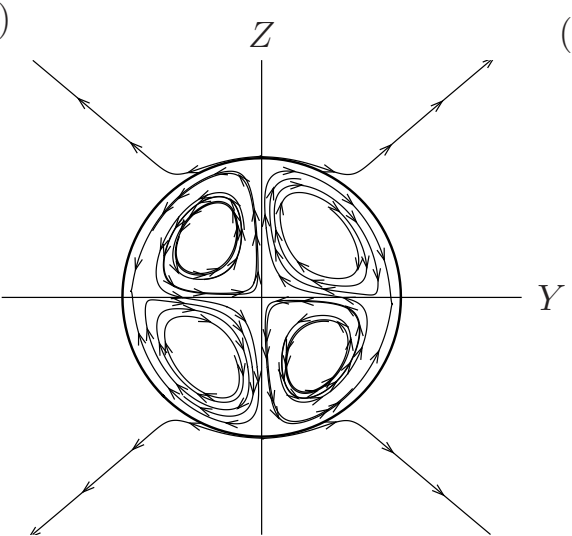

(b)

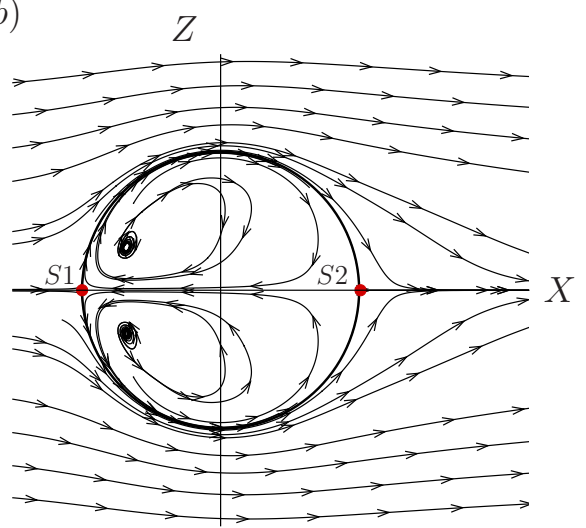

$(d)$

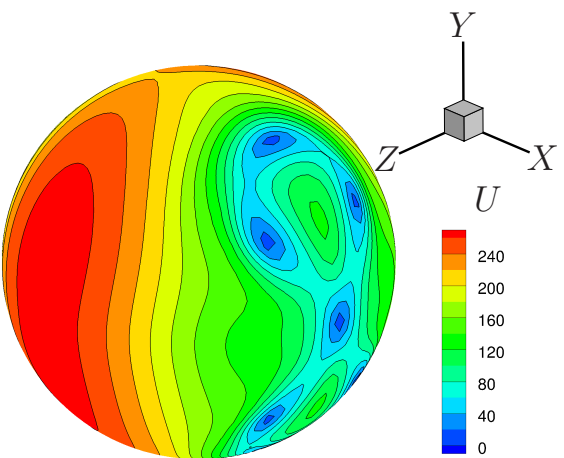

Figure 8: Streamlines $(\mathrm{a}-\mathrm{c})$ inside and outside of a fluid sphere as well as contours of absolute velocity (d)) at the sphere surface for $R e=300, \lambda=0.5, \kappa=1.5$. In a - c, planes through the origin are shown. Red dots indicate stagnation points in the outer flow, denoted by $S 1$ and $S 2$.

At $R e=400$, the solution is highly unsteady and different modes of internal flow structures can be observed. However, the detailled study of these unsteady phenomena is beyound the scope of the present paper. Furthermore, complicated interactions of the inner flow field with both velocity changes in the outer flow field and interface deformations are possible, which cannot be captured with the present method. For a study where these phenomena are considered, see Engberg and Kenig (2015). 


\subsection{Drag in Liquid-Liquid Systems}

In this section, the influence of the different modes of internal circulation (described in the last section) on the drag is discussed. As the outer Reynolds number influences the drag coefficient even if the internal circulation is identical, the inner Reynolds number is changed instead. This is done by changing $\kappa$, while all other parameters are kept constant. For two-dimensional, axisymmetric simulations (see e.g. Feng and Michaelides (2001)), $\kappa$ has only a minor influence on the drag. However, in our simulations a varying $\kappa$ may significantly change the flow both inside and outside of the drop and should therefore influence the drag in those cases.

We consider the case at $R e=200, \lambda=0.5$ and vary $\kappa$ from 0.25 up to 1.8. The drag coefficient for these cases as well as for those discussed in the last section can be found in Table 6 . Furthermore, some cases were simulated with a finer

mesh (see section 2 for details on the meshes) to show the grid independence of the results. At both $\kappa=0.25$ and $\kappa=0.75$, a Hill's vortex-like solution, similar to the one shown in Figure 5 is obtained. In accordance with the literature, only minor differences in drag between both cases are visible. For $\kappa=1.125$ and $\kappa=1.2, R e_{i}$ is near the $R e_{i}$ value found for the $R e=150, \lambda=0.5, \kappa=1.5$ case. In these cases, a solution with one plane of symmetry is found, similar to the one visualized in Figure 6. Compared with the Hill's vortex-like solutions, the drag is greatly increased ( $>30 \%$ ), which is mainly due to an increase in pressure drag. Furthermore, even with similar internal structures, $\kappa$ influences the drag coefficient, as the increasing $\kappa$ from 1.125 to 1.2 increases the drag by roughly $2 \%$. The last two cases studied $(\kappa=1.5$ and $\kappa=1.8)$, both exhibit a solution with two planes of symmetry as shown in Figure 7. Again, the drag is increased compared to the aforementioned cases, mainly due to an increase in pressure drag. Furthermore, the $20 \%$ increase in $\kappa$ and thus in $R e_{i}$ between the 
Table 6: Drag coefficients for viscous spheres in the liquid-liquid regime.

\begin{tabular}{cccccccc}
\hline \multirow{2}{R}{$\lambda^{*} \lambda$} & \multirow{2}{*}{$R_{i}$} & \multicolumn{3}{c}{$C_{D}$} & $C_{D, f}$ & $C_{D, p}$ \\
& & & & Mesh 1 & Mesh 2 & & \\
\hline 50 & 0.5 & 1.5 & - & 0.920 & - & 0.536 & 0.384 \\
100 & 0.5 & 1.5 & 291 & 0.538 & - & 0.309 & 0.229 \\
200 & 0.5 & 0.25 & 112 & 0.310 & - & 0.175 & 0.135 \\
200 & 0.5 & 0.75 & 330 & 0.306 & 0.307 & 0.170 & 0.136 \\
\hline 150 & 0.5 & 1.5 & 431 & 0.492 & - & 0.264 & 0.228 \\
200 & 0.5 & 1.125 & 453 & 0.410 & 0.409 & 0.214 & 0.196 \\
200 & 0.5 & 1.2 & 478 & 0.419 & - & 0.217 & 0.202 \\
\hline 200 & 0.5 & 1.5 & 554 & 0.466 & 0.465 & 0.235 & 0.231 \\
200 & 0.5 & 1.8 & 634 & 0.492 & - & 0.247 & 0.246 \\
250 & 0.5 & 1.5 & 682 & 0.442 & - & 0.210 & 0.231 \\
\hline 300 & 0.5 & 1.5 & 818 & 0.421 & - & 0.191 & 0.230 \\
\hline
\end{tabular}

case with $\kappa=1.5$ and the one with $\kappa=1.8$ leads to a further increase in drag of approximately $5.5 \%$, .

To summarize, in our full three-dimensional simulations, the density ratio has a major influence on the drag, as soon as the Hill's vortex-like solution disappears. In the simulation for liquid-liquid systems, cases with similar $R e_{i}$ showed similar inner flow structures. However, this finding should not be extrapolated to arbitrary cases with substantial differences in $R e$ and $\lambda$, as the outer flow field influences the inner flow field and furthermore only a limited parameter range was studied.

In Figure 9, our present results are compared with results from the literature. On the one hand, Feng and Michaelides (2001) conducted two-dimensional, axisymmetric numerical simulations of cases at $\lambda=0.5(\kappa=0.5)$ and developed a correlation function for viscous spheres which is valid for $0 \leq \lambda \leq 2$. (They also 
developed a function for $2 \leq \lambda \leq \infty$.) On the other hand, Thorsen et al. (1968) performed experiments with ethyl bromide drops falling in water $(\lambda=0.53$, $\kappa=1.45)$. It can be seen that at $R e=50$ and $R e=100$ the present results are very close to the numerical results from Feng and Michaelides (2001), whereas the results deviate at higher Reynolds numbers due to the different internal flow modes. The correlation function for viscous spheres is slightly off with respect to the numerical results by Feng and Michaelides (2001) but follows the general trend for the Hill's vortex-like cases. Even though the experimental case at $R e=170$, as well as the present simulation results at $R e=150$, are very close to the correlation function, this seems to be by chance, as the function generally overestimates the drag for the cases at $\lambda=0.5$ from Feng and Michaelides (2001). The correlation function for rigid spheres approaches the function for viscous spheres at low Reynolds numbers but diverges from all other results for higher Reynolds numbers. It is evident from Figure 9 that the experimental results by Thorsen et al. (1968) are close to the present results but (at least at higher Reynolds numbers) far from the results by Feng and Michaelides (2001). This supports the idea that the assumption of cylindrical symmetry is not valid in these cases and that our method is able to correctly reproduce the physical phenomena.

\subsection{Flow Structures in Liquid-Gas Systems}

For the discussion of liquid drops in a uniform gas stream, cases with low viscosity ratio and high density ratio are searched, similar to the cases in liquidliquid systems (see section 5.1). As the viscosity of most liquids decreases with increasing temperature, whereas the viscosity of gases displays the opposite behaviour, high temperatures are beneficial. However, at higher temperatures, evaporation becomes important (emerging of a vapor boundary layer and Stefan flow), which means that our method in no longer valid. Thus, we begin our dis- 


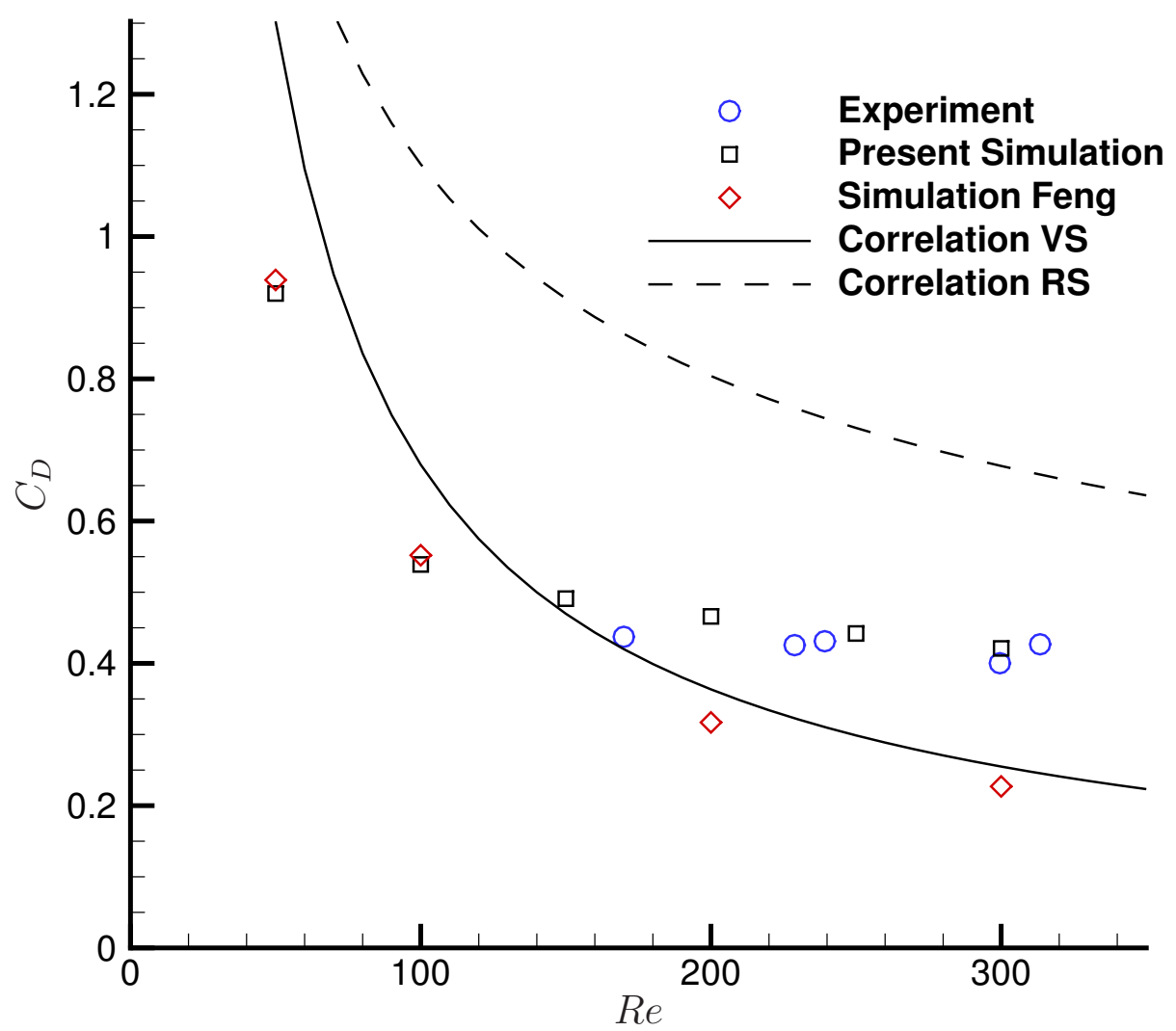

Figure 9: Drag coefficient versus Reynolds number for ethyl bromide drops in water. Experiment: Data by Thorsen et al. (1968). Present Simulation: Results at $\lambda=0.5, \kappa=1.5$. Simulation Feng: Two-dimensional axisymmetric simulations at $\lambda=0.5, \kappa=0.5$ from Feng and Michaelides (2001). Correlation VS: Correlation for viscous spheres from Feng and Michaelides (2001). Correlation RS: Correlation for rigid spheres used by Feng and Michaelides (2001), originally by Putnam (1961). The equations for the correlations are given in Appendix A. 
cussion with a case at $\lambda=20 \kappa=600$, which approximates n-heptane drops in air at $30^{\circ} \mathrm{C}$ and 1 bar, where the vapor pressure is roughly 0.08 bar. In order to arrive at similar internal Reynolds numbers as for the liquid-liquid systems, we start at $R e=100$. From the literature (see e.g. Waheed (2001) p.p. $77-78$ for cases at $R e=100$ and $R e=200$ with $\lambda=10$ and $\kappa=1$ ) a distinct recirculation zone behind the drop is expected. In Figure 10, streamlines inside and outside the drop in three planes through the origin, as well as the absolute velocity on the drop surface are shown for $R e=100, \lambda=20, \kappa=600$. A typical Hill's vortex-like solution can be seen inside the drop. Outside, a steady, cylindrical symmetric recirculation zone is visible. This trailing vortex is detached from the drop because the flow directly adjacent to the drop surface stays attached to the drop due to the internal circulation. Four stagnation points, denoted by $S 1-S 4$ are found. As the outer recirculation zone is detached from the drop, $S 2$ and $S 3$ are not identical but less than $0.02 d$ apart.

In the same way as for the liquid-liquid systems, the Reynolds number is increased to 150 , whereas $\kappa$ and $\lambda$ remain constant. The flow field for that case is visualized in Figure 11. Similar to the case at $R e=300, \kappa=0.5, \lambda=1.5$, shown in Figure 8, the solution is unsteady with minimal fluctuations and no plane of symmetry, but a two-fold rotational symmetry around the $x$-axis is found. In the $x-y$ plane, the outer flow separates from the drop which means that the trailing vortex is now attached to the drop in this plane. Note that the two stagnation points, one in the upper and one in lower half, are not shown as they are slightly off of the plane. However, in the $x-z$ planes the outer flow stays attached to the drop (thus the trailing vortex is detached from drop) which means that a stagnation point (denoted by $S 3$ ) exists which is only $\approx 0.01 d$ apart from the rear stagnation point $(S 2)$ at the surface.

When comparing the results for liquid-gas systems with those for liquid-liquid 
(a)

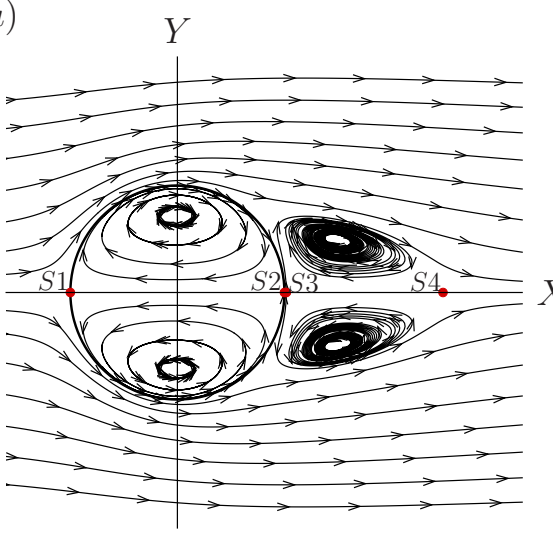

$(c)$

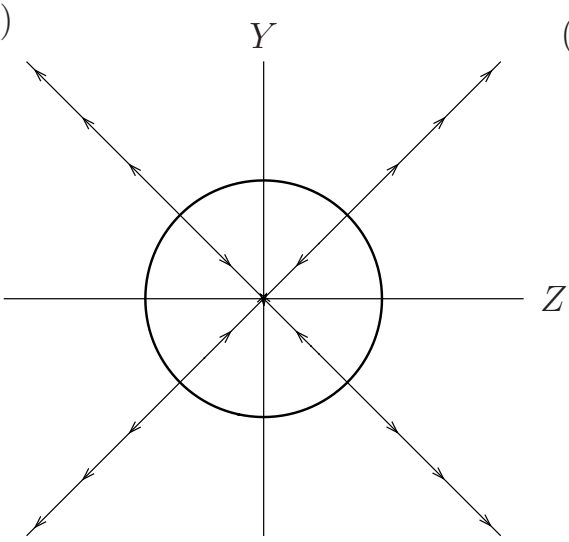

(b)

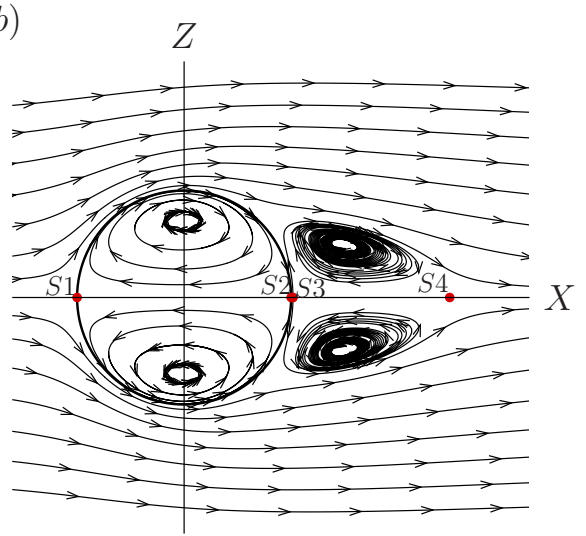

$(d)$

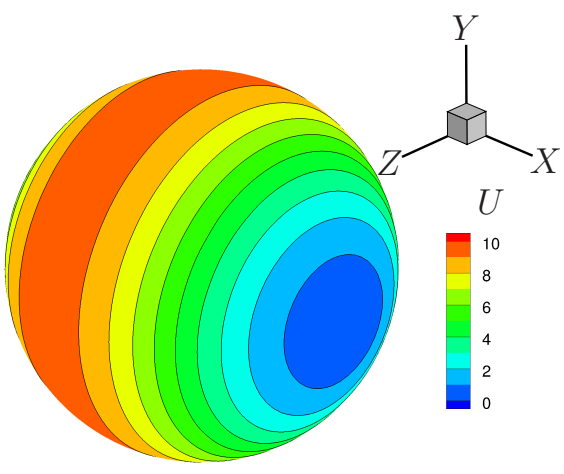

Figure 10: Streamlines $(\mathrm{a}-\mathrm{c}$ ) inside and outside of a fluid sphere as well as contours of absolute velocity (d) at the sphere surface for $R e=100, \lambda=20, \kappa=600$. In a - c, planes through the origin are shown. Red dots indicate stagnation points in the outer flow, denoted by $S 1, S 2, S 3, S 4$. 
(a)

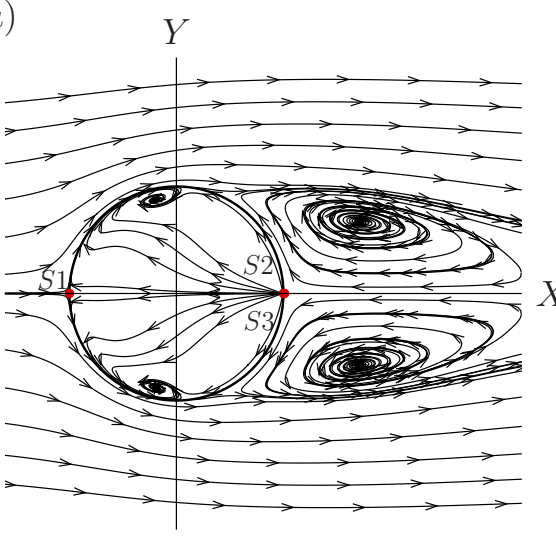

$(c)$

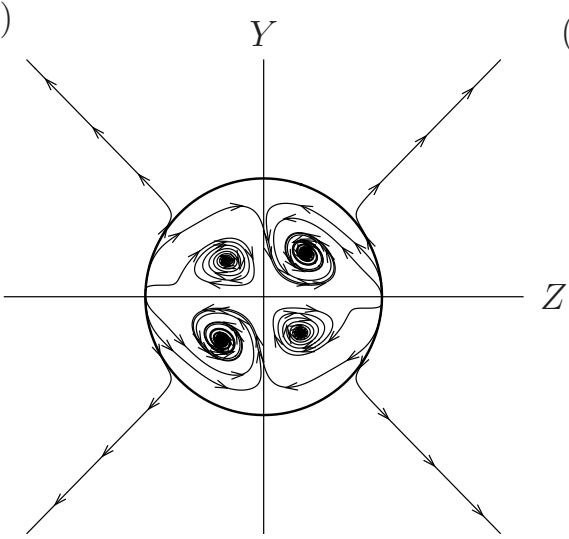

(b)

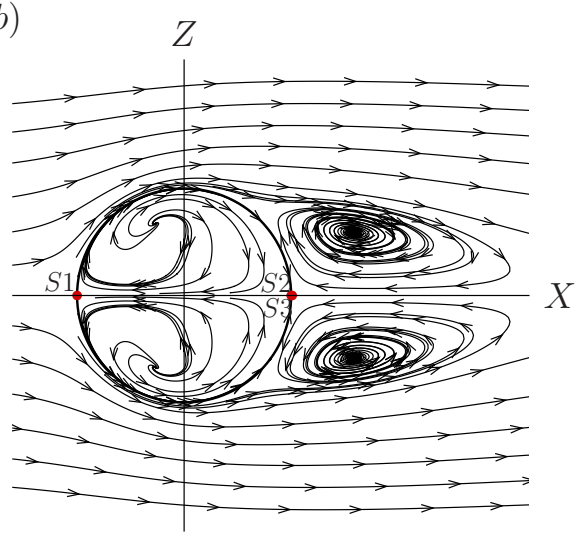

$(d)$

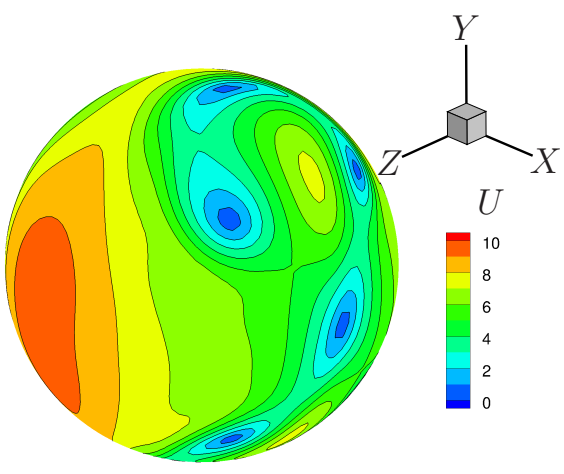

Figure 11: Streamlines $(\mathrm{a}-\mathrm{c})$ inside and outside of a fluid sphere as well as contours of absolute velocity (d) at the sphere surface for $R e=150, \lambda=20, \kappa=600$. In a - c, planes through the origin are shown. Red dots indicate stagnation points in the outer flow, denoted by $S 1$ and $S 2$. 
systems, it seems that somehow two solutions for the internal flow structures (the case with one symmetry plane and the case with two symmetry planes) are missing. Therefore, a case with $R e=130, \lambda=20, \kappa=600$ was simulated. However, even in this case, the solution resembles the one shown in Figure 11. Subsequently, a case with $R e=100$ (keeping $\lambda$ and $\kappa$ constant) was simulated but started from the converged results at $R e=150$ (flow field shown in Figure 11). Instead of switching back to the cylindrical symmetric solution shown in Figure 10, a solution similar to the case at $R e=150$ is obtained. However, in contrast to the case at $R e=150$, two planes of symmetry, the $x-y$ and the $x-z$ plane, are found. As the flow field is very similar to the one shown in Figure 11 it is not visualized. To check the stability of both solutions found at $R e=100$, a sudden velocity perturbation was added to the stationary solution inside the drop and the simulations were run until a new stationary solution was reached. A moderate disturbance (with amplitude $\approx 0.1 U_{i}$ ) and a severe disturbance (with amplitude $\approx U_{i}$ ) were researched. For a moderate disturbance, both modes remained stable. However, for the severe disturbance, in both cases the solution with two planes of symmetry was obtained. It therefore seems that for the same set of parameters one stable and one conditionally stable solution are found. If the Reynolds number is further reduced to 50 (started from the converged results at $R e=150$ ) the resulting flow field is again cylindrical symmetric with a Hill's vortex-like internal solution.

We proceed by increasing $\kappa$, whereas the $R e$ and $\lambda$ remain at 50 and 20, respectively. Up to $\kappa=1,800$, a cylindrical symmetric solution with a Hill's vortexlike internal motion is obtained. At $\kappa=2,400$, the asymptotic flow field solution is again two-fold rotational symmetric around the $x$-axis (with very small fluctuations), with the internal flow structures resembling the structures shown in Figure 11. Three additional simulations at $R e=50, \lambda=20$ were performed 
with $\kappa=1,800, \kappa=1,200$ and $\kappa=120$. They were started from the converged solution at $\kappa=2,400$ and simulated until a new converged solution was found. The flow structures at $\kappa=1,800$ and $\kappa=1,200$ resemble the structures of the case with $\kappa=2,400$ (see Figure 11), whereas at $\kappa=1,200$ a Hill's vortex-like internal motion is observed. Even though the above mentioned cases may be unphysical, they show that the initial conditions of the simulation are of great importance. Furthermore, the solution with two symmetry planes obtained for a liquid drop in a liquid (see Figure 7) was, for a liquid drop in a gas, only found if the internal Reynolds number was reduced from a converged case with four vortex structures in the $y$ - $z$ plane and a two-fold rotational symmetry around the $x$-axis (see Figure 11).

In all aforementioned cases in liquid-gas systems, an outer recirculation zone is obtained, which is not the case in the liquid-liquid cases studied. This is due to the much higher surface velocities for drops in a liquid, as the viscosity ratio is lower. However, another possibility to avoid the formation of the trailing separation zone is to lower the outer Reynolds number, as even for a rigid sphere the flow stays attached until roughly $R e=20$ (see Johnson and Patel (1999)). As this value is higher for a viscous sphere, we simulated cases at $R e=25$. $\lambda$ was held constant at 20 and the internal Reynolds number was varied by changing $\kappa$. At $\kappa=3,600$ and $\kappa=4,800$, a Hill's vortex-like solution inside the drop is found ${ }^{3}$, whereas the flow outside remains attached.

At $\kappa=5,400$, the cylindrical symmetric solution disappears and a solution with four vortical structures inside the drop in the $y$ - $z$ plane and a two-fold rotational symmetry around the $x$-axis is found, as visualized in Figure 12 . The asymp-

\footnotetext{
${ }^{3} \mathrm{~A}$ close examination of the residuals reveals that the case at $\kappa=4,800$ might for very large times switch to a different mode. Even though this case was run longer than any other case in the present study, this would probably take more than twice as long (more than another $10,000 \mathrm{cpu}$ hours on our present system).
} 
totic solution is quasi-steady and the flow field inside the drop is very similar to the already discussed case at $R e=150, \kappa=600$, even though the parameters are quite different. However, the outer flow is very dissimilar, which is best seen in the $x$-y plane (Figure 12 a). Even though the flow separates quite early (visualized by the red dots), no recirculating trailing vortex is found. This is similar to the cases in the liquid-liquid regime (see e.g. Figure 8). In contrast to these cases however, only a minimal widening of the streamlines is observed in the wake region.

Two additional cases, one with $\kappa=6,000$ and one with $\kappa=7,200$ were simulated. The flow field increasingly deviates (with increasing $\kappa$ ) from the plane symmetric solution, similar to the behavior studied in the liquid-liquid regime (see Figure 8). Furthermore, the oscillation amplitude of the unsteady motion is increasing with increasing $\kappa$. As the flow field for both cases is in other aspects not very different from the aforementioned case at $\kappa=5,400$, it is not visualized.

The last case studied is a water drop in air at $R e=300, \lambda=49, \kappa=829$ as investigated by Sugioka and Komori (2007) and briefly mentioned in section 4. In this case, Sugioka and Komori (2007) found a quasi-steady flow field which is plane symmetric but not axisymmetric. As we were not able to find such a case for $\lambda=20$, we will discuss the differences in the following. A more detailed discussion of that case can be found in Sugioka and Komori (2007). As can be seen from Figure 13, some flow features are remarkably different to the aforementioned cases. First of all, the trailing vortex is itself not axisymmetric. Furthermore, inside the drop, only one pair of vortical structures can be found in the $y$ - $z$ plane. Most important however, the internal flow in both the $x-y$ and the $x-z$ plane looks very much like a Hill's vortex and no reversal of the flow direction on the drop surface is observed. It thus seems that the alteration of 
(a)

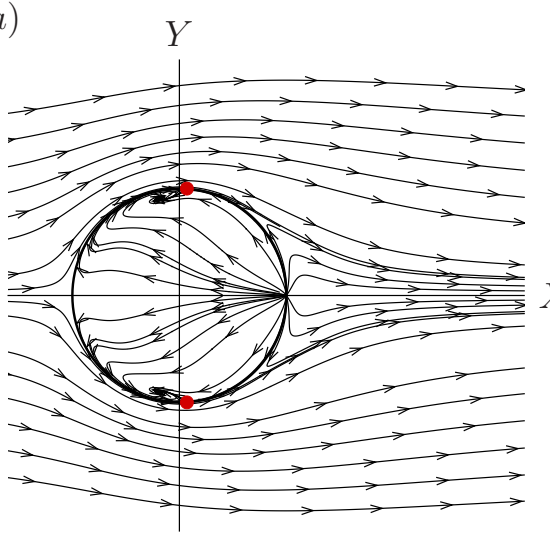

$(c)$

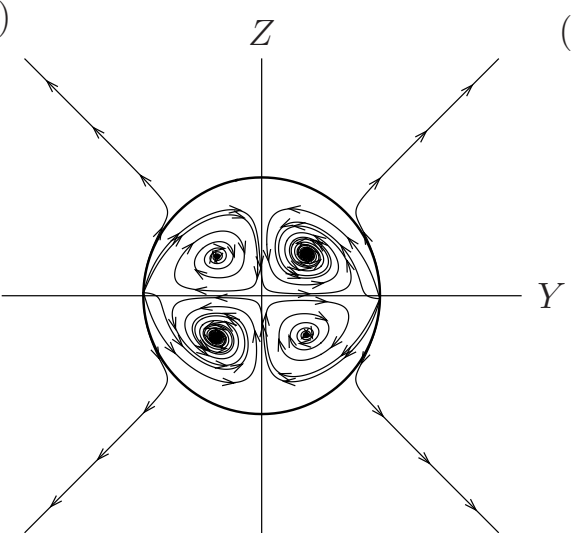

(b)

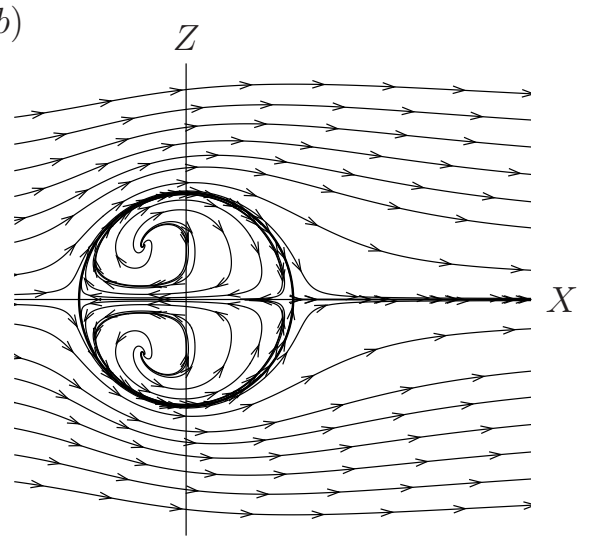

$(d)$

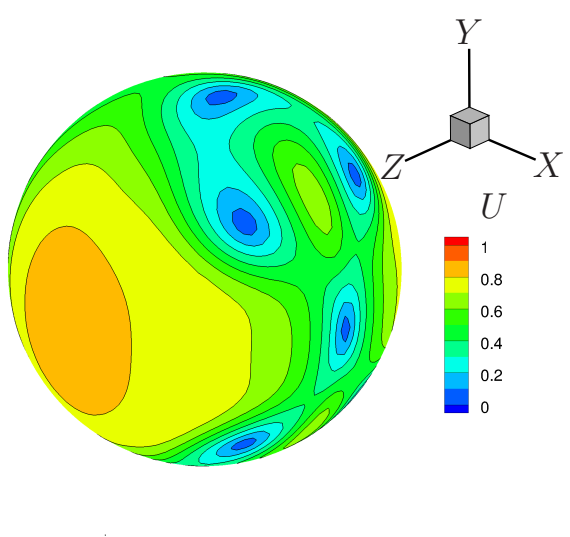

Figure 12: Streamlines $(\mathrm{a}-\mathrm{c})$ inside and outside of a fluid sphere as well as contours of absolute velocity (d) at the sphere surface for $R e=25, \lambda=20, \kappa=5,400$. In a - c, planes through the origin are shown. The red dots denote the points of flow separation in the $x-y$ plane. 
$(a)$

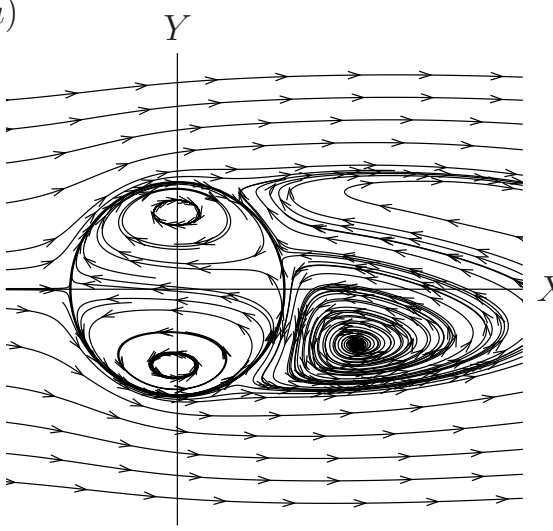

(c)

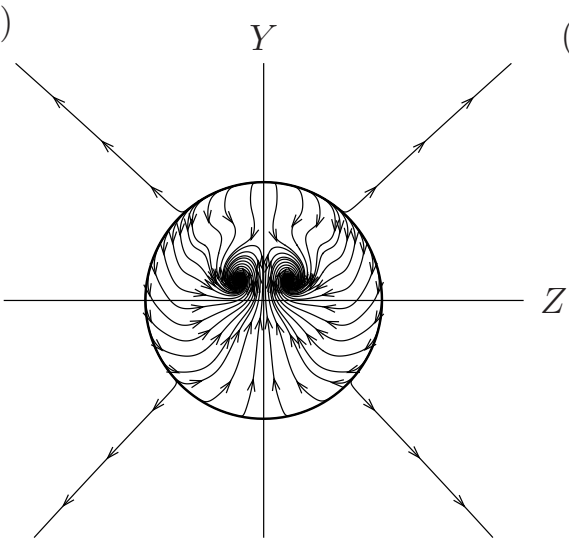

(b)

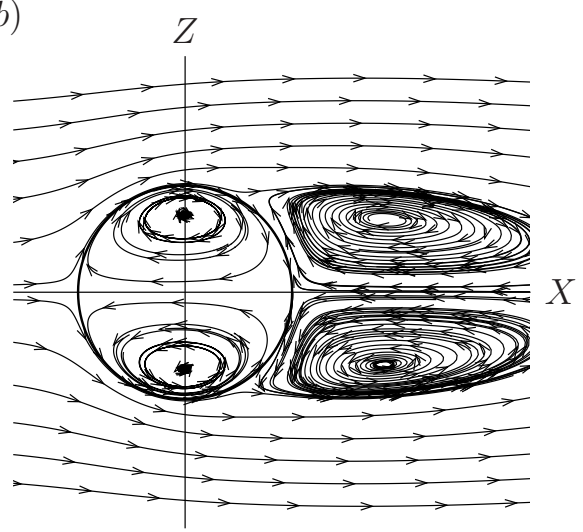

$(d)$

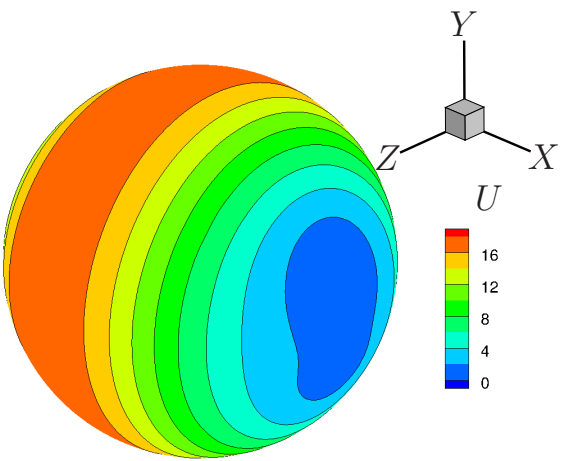

Figure 13: Streamlines $(\mathrm{a}-\mathrm{c})$ inside and outside of a fluid sphere as well as contours of absolute velocity (d) at the sphere surface for $R e=300, \lambda=49, \kappa=829$ (water drop in air). In a - c, planes through the origin are shown.

the Hill's vortex is in this case due to the non-axisymmetric trailing vortex and not because of an internal flow instability.

\subsection{Drag in Liquid-Gas Systems}

In Table 7, drag coefficients for the cases discussed in the last section are presented. For two cases, $R e=150, \lambda=20, \kappa=600$ and $R e=300, \lambda=49$, $\kappa=829$, a close agreement of results on Mesh 1 and Mesh 2 (see section 3) is demonstrated. In the upper part of Table 7, cases which exhibit a Hill's vortexlike internal solution are shown, whereas in the lower part, cases with four 
vortical structures in the $y-z$ plane and two-fold rotational symmetry around the $x$-axis and four vortical structures in the $y$ - $z$ plane are presented. The two cases in the middle are the case at $R e=300$ with one symmetry plane and the case at $R e=25$ with two symmetry planes. By comparing cases with identical $R e$ and $\lambda$ it becomes obvious that a change in density ratio has nearly no influence on drag, whether the internal circulation mode changes or not. This is due to the relatively slow velocities on the surface compared to free stream values. Even the reversal of the flow direction in the rear part of the drop is not able to significantly change the outer flow field and thus the form drag, a fact illustrated in Figure 11 and Figure 12. Furthermore, as the velocities on the surface are so slow, the drag coefficient is in all cases studied (in the liquid-gas regime) very close to the drag of a rigid sphere at the same Reynolds number (see Table 3 and Feng and Michaelides (2001) for comparison).

In contrast to the cases in the liquid-liquid regime (see section 5.2), the inner Reynolds number does not seem to be a good indicator for the mode of internal flow, as $R e_{i}$ sharply decreases if the internal flow field diverges from the Hill's vortex-like solution. The velocities at the drop surface are quite small compared with the outer flow. However, when the internal flow mode changes, the relative difference is quite large. For example, at $R e=25, \lambda=20$, the maximum absolute velocity at the surface decreases by approximately $40 \%$ when $\kappa$ is increased from 4,800 to $\kappa=5,400$. Furthermore, as shown in section 5.3, the initial condition is of importance. For the same set of simulation parameters $(R e, \lambda, \kappa)$, different flow fields and internal Reynolds numbers may be found.

To conclude, the present calculations show that the drag coefficient for viscous spheres in the liquid-gas regime depends in first order only on the Reynolds number of the outer fluid. For the state of the internal flow, a bifurcation with (at least) two solutions was found. On the one hand, identical inner Reynolds 
numbers might be obtained for a different set of simulation parameters. On the other hand, identical simulation parameters but different initial conditions, might lead to different flow fields and thus different internal Reynolds numbers.

\subsection{Internal Problem of Heat/Mass Transfer}

In this section, the influence of the different modes of internal circulation on the heat/mass transfer inside the drop will be discussed. For cases with a Hill's vortex-like internal flow (see e.g. Figure 10) a fluid particle inside a drop always stays on a two-dimensional, nearly ellipsoidal path. However, when that cylindrical symmetry is lost (see e.g. Figure 7), a fluid particle inside a drop has a much more complicated, three dimensional path. Thus, at least for high Peclet numbers, it is expected that the mode of internal circulation has a major influence on the internal transfer. In the following, we concentrate on the internal problem of heat/mass transfer, which assumes that the main transfer resistance is located inside the drop. This implies that the transfer in the outer phase is much faster and the concentration on the interface is constant (see e.g. Wegener et al. (2014)). Especially in cases with locally high Sherwood/Nusselt numbers (high mass/heat flux), the assumption of an internal problem might be invalid. The correct classification, however, depends on diffusion and concentration ratios between inner and outer fluid, which are undefined in the present study.

For the present study, very high Peclet numbers up to $P e_{i}=10,000$ are considered. As these values are higher than those studied for the basic validation (section 4), a grid study was performed for some cases (for details on the

meshes see section 3$)$. The asymptotic Sherwood numbers $(t \rightarrow \infty)$ for these cases can be found in Table 8. At $R e=150, \lambda=20, \kappa=600$, no stationary 
solution for the flow field was observed, thus the asymptotic Sherwood numbers are also unsteady (indicated by a "+" in Table 8). In these cases, the average value of the last oscillation was taken as the asymptotic value. It can be seen from the table that for most cases, $S h_{\infty}$ from simulations on different meshes is in good agreement. However, for $R e=150, \lambda=0.5, \kappa=0.75$ (a case with a Hill's vortex-like internal flow field) at $P e_{i}=5279$ and $P e_{i}=10559, S h_{\infty}$ from simulations on Mesh 1 and Mesh 2 deviate by roughly $3 \%$ and $6 \%$, respectively. In Figure 14, asymptotic Sherwood numbers are plotted versus Peclet number for cases at $R e=200$ and $\lambda=1.5$ (liquid - liquid regime) with varying $\kappa$ (flow structures for these cases are described in section 5.1). At $\kappa=0.75$, where a Hill's vortex-like internal flow field is observed, a $S h_{\infty}$ value of around 20 is found for high $P e_{i}$, which is in agreement with preceeding studies (e.g. Juncu (2010)). At very high $P e_{i}$, it seems that the Sherwood number is slightly increasing. However, it was shown above that this is most likely an artifact of the insufficient spatial resolution (see Table 8). At $\kappa=1.125$ and $\kappa=1.2$, a solution without axisymmetry but with one plane of symmetry is found (see Figure 6). Compared with the Hill's vortex-like internal solution, at moderate Peclet numbers up to roughly $P e_{i}=200$ lower $S h_{\infty}$ values are observed, whereas at higher Peclet numbers higher values are obtained. At $\kappa=1.5$ and $\kappa=1.8$, where a solution with two symmetry planes is found, the behavior is similar to the aforementioned case. However, for moderate Peclet numbers, $S h_{\infty}$ is even lower, whereas it is higher for high Peclet numbers, exceeding the Hill's vortex cases at $P e_{i} \approx 300$ and the one plane symmetric case at $P e_{i} \approx 1,000$. Interestingly, $S h_{\infty}$ for the non-cylindrical symmetric cases keeps increasing exponentially even for very high $P e_{i}$. If only values with $P e_{i} \geq 2000$ are taken, a least squares fit yields an exponent of 0.26 for the cases with one symmetry plane and an exponent of 0.37 for the cases with two symmetry planes. 


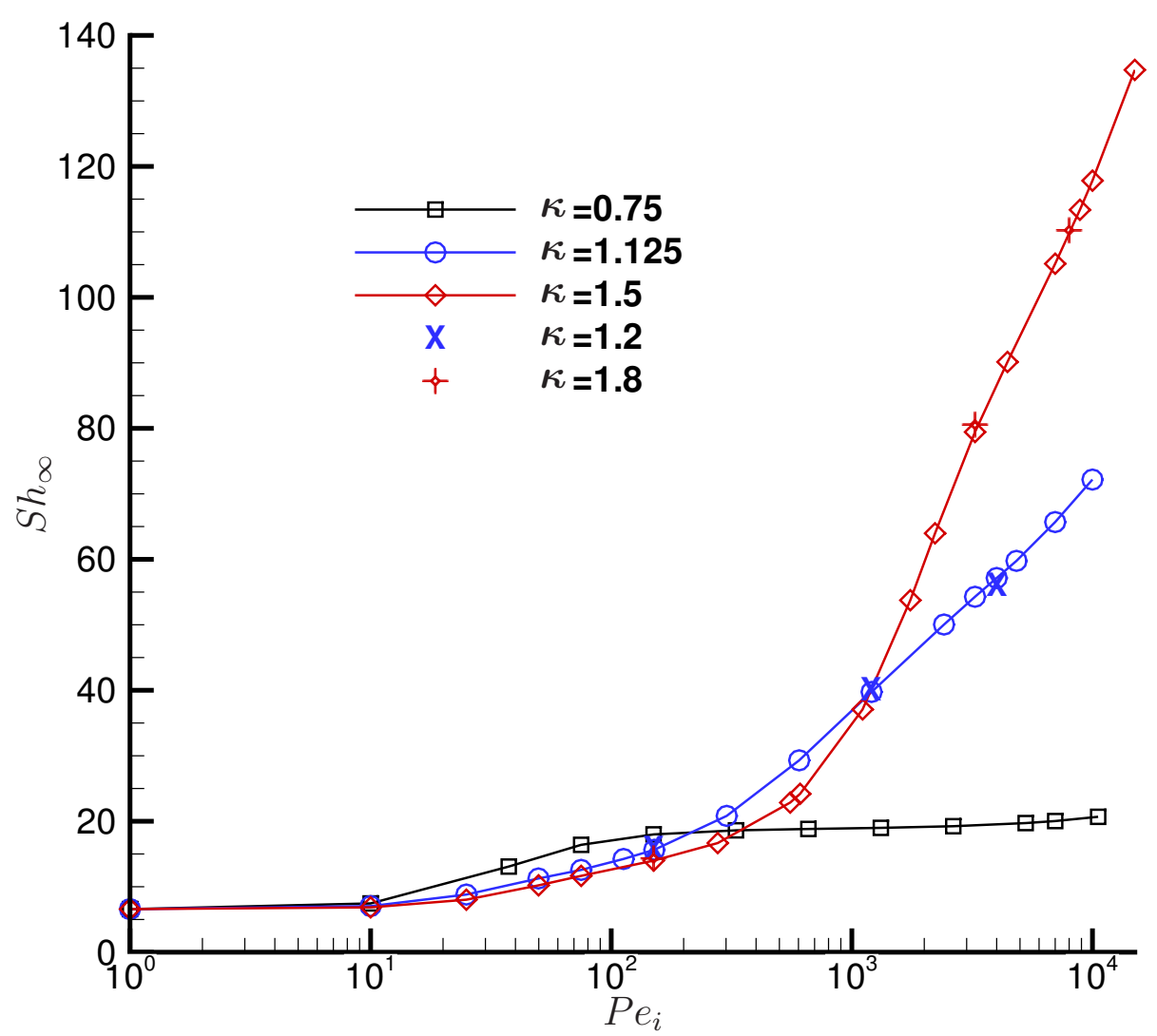

Figure 14: Asymptotic Sherwood number versus Peclet number at $R e=200$ and $\lambda=1.5$ with varying $\kappa$ (liquid-liquid regime).

Asymptotic Sherwood numbers versus Peclet number for liquid-gas systems are plotted in Figure 15. Similar observations as in the before discussed Figure can be made. $S h_{\infty}$ from cases with two-fold rotational symmetry ${ }^{4}$ exceed the values from the Hill's vortex-like solution for roughly $P e_{i}>200$ and keep increasing in the parameter range studied. Even though the parameters are very different, $S h_{\infty}$ values from cases with two-fold rotational symmetry (case $R e=150, \lambda=20, \kappa=600$ and case $R e=25, \lambda=20, \kappa=5,400)$ are very close. Compared to the cases with two symmetry planes in the liquid-liquid regime

\footnotetext{
${ }^{4}$ Note that in those cases where the flow field is unsteady, no truly asymptotic Sherwood number can be found. Instead the average value of the last oscillation studied is taken.
} 


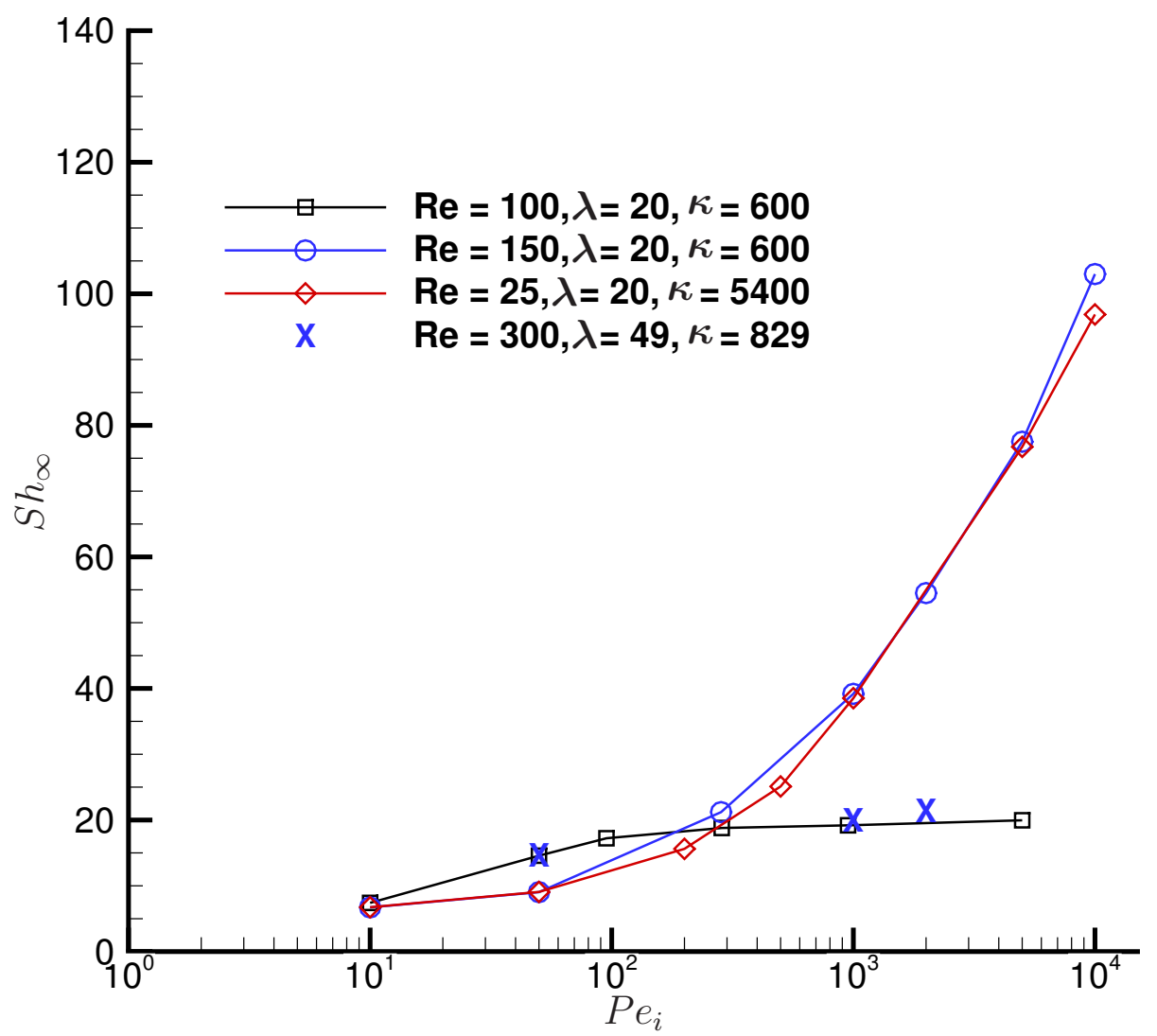

Figure 15: Asymptotic Sherwood number versus Peclet number for cases in the liquid-gas regime. At $R e=100, \lambda=20, \kappa=600$, results for a case with a Hill's vortex-like internal solution are presented.

(e.g. $R e=200, \lambda=0.5, \kappa=1.5$ ), a similar slope is observed, but values are lower at high Peclet numbers above $\approx 1,000$ and inversely below. For high $P e_{i}$ an exponential growth is obtained. A least squares fit for $P e_{i} \geq 2000$ gives an exponent of 0.38 , which is only slightly above the value of 0.37 for the cases with two symmetry planes in the liquid-liquid regime. For the water droplet in air at $R e=300(\lambda=49, \kappa=829), S h_{\infty}$ values are only slightly above the Hill's vortex-like solution.

By comparing only asymptotic Sherwood numbers, the time evolution of the mass/ heat transfer is ignored, which might be important as time oscillations 
are even observed for the Hill's vortex internal solution (see section 4). The instantaneous Sherwood number is plotted versus nondimensional time in Figure 16 and Figure 17 for the liquid-liquid and the liquid-gas regime, respectively. At low Peclet numbers, Sherwood numbers are nearly identical at small times. However, at larger times, where $S h$ is again increasing, slopes for the cases with a Hill's vortex-like inner flow field (case $\kappa=0.75$ in Figure 16 and $R e=100$ in Figure 16) lie above those with non-cylindrical symmetric solutions. At very high Peclet numbers, it can be seen that the Sherwood numbers, for different internal flow field solutions, are in the same order of magnitude only at very small times. For the cases shown, the $S h$ values diverge from one another quickly at approximately $t^{\prime}=2 \cdot 10^{-3}$.

An important parameter to model the transfer inside a drop is the (nondimensional) saturation time $\left(t_{\text {sat }}^{\prime}\right)$. In the present study, the saturation time is defined as the time until the average concentration $(\tilde{c})$ inside the drop has reached 0.99. The saturation time is plotted versus the internal Peclet number in Figure 18 and Figure 19 for the liquid-liquid and the liquid-gas regime, respectively. In the diffusion limit (in the present study $P e=1$ was used), $t_{\text {sat }}^{\prime} \approx 0.419$ in all cases, which is in good agreement with the theoretical value, which is $t_{\text {sat }}^{\prime}=0.416$ for $P e=0$. For high values of $P e_{i}$, the huge differences between cases with (i.e. Hill's vortex-like cases) and without cylindrical symmetry get obvious. For cases with a Hill's vortex-like internal flow structure $(\kappa=0.75$ in Figure 18 and $R e=100, \lambda=20, \kappa=600$ in Figure 19$), t_{\text {sat }}^{\prime} \approx 0.14$ for high $P e_{i}{ }^{5}$, whereas no asymptotic value for $t_{\text {sat }}^{\prime}$ was found for high $P e_{i}$ for the non-cylindrical symmetric cases in the parameter range studied. The lowest $t_{\text {sat }}^{\prime}$ obtained in the present study was $t_{\text {sat }}^{\prime}=0.023$ for $P e_{i}=15,000, R e=200$,

\footnotetext{
${ }^{5}$ Note that the decrease of the saturation time for high Peclet numbers for cases with a Hill's vortex-like internal flow are probably due to an insufficient spatial resolution. This is similar to the behavior shown in Figure 14 and discussed at the beginning of this section
} 


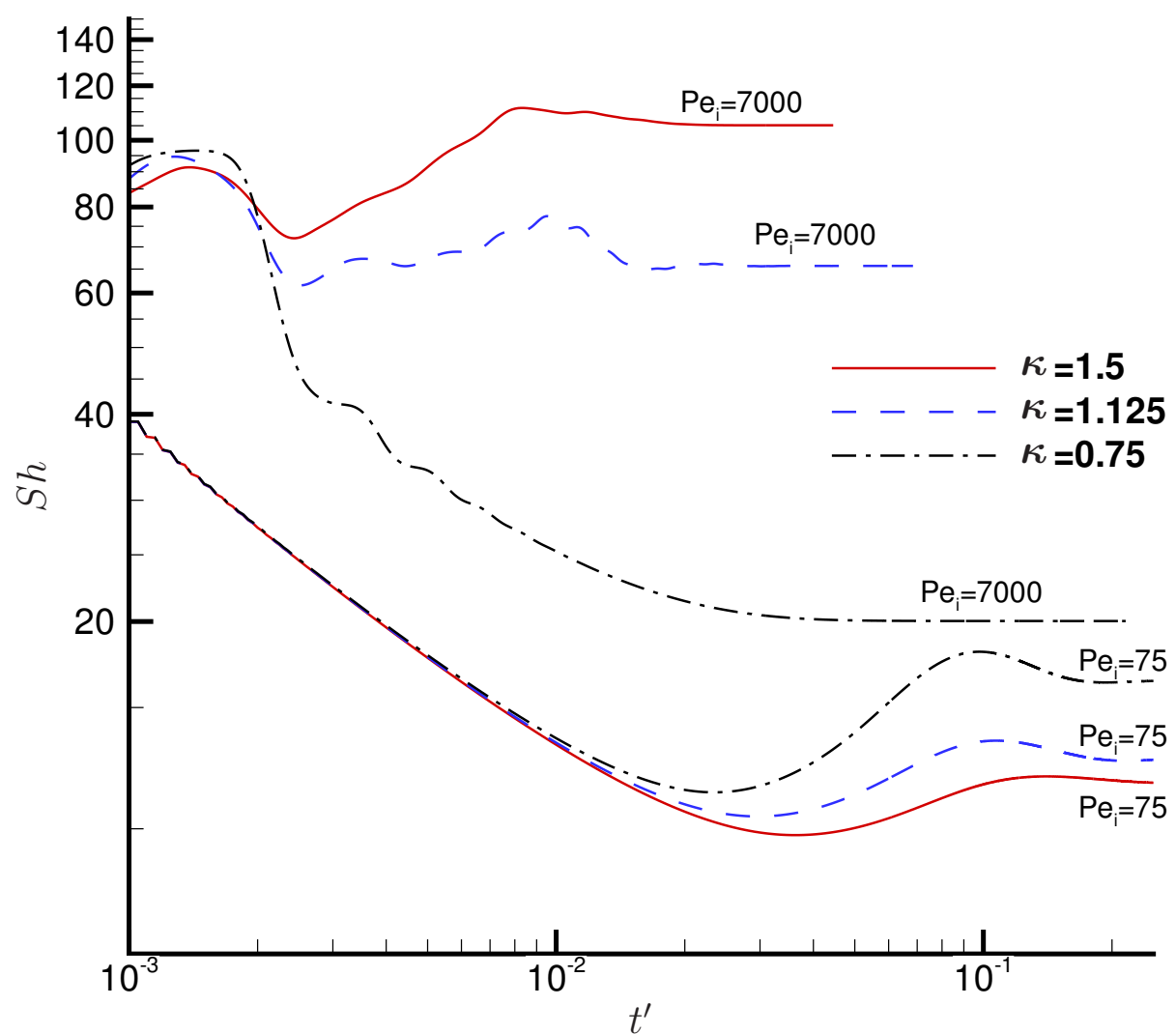

Figure 16: Instantaneous Sherwood number versus nondimensional time $t^{\prime}$ at $R e=200$ and $\lambda=1.5$ with varying $\kappa$ and $P e$ (liquid-liquid regime). 


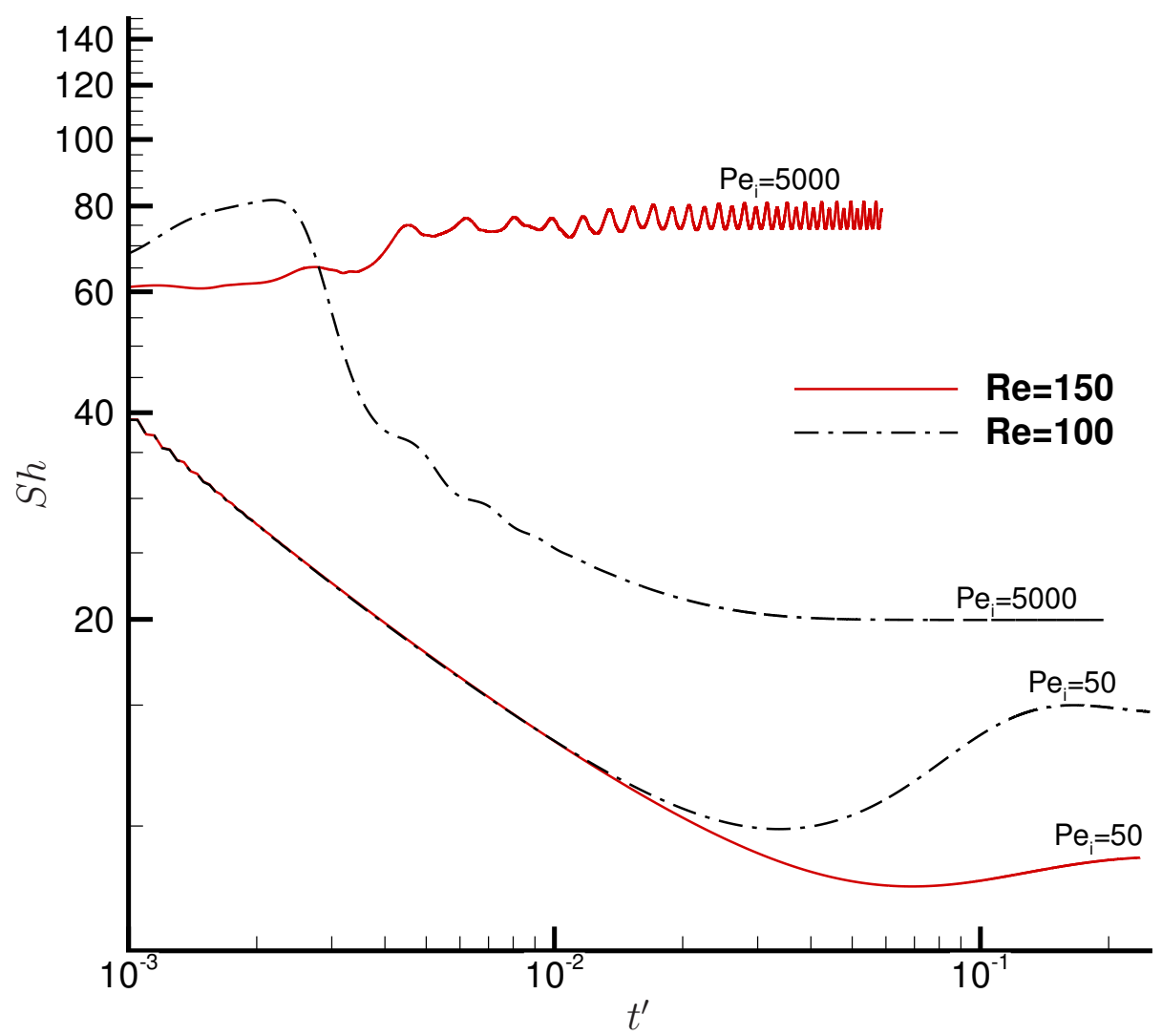

Figure 17: Instantaneous Sherwood number versus nondimensional time $t^{\prime}$ at $\lambda=20$ and $\kappa=600$ with varying $R e$ and $P e$ (liquid-gas regime). At $R e=100$, results for a case with a Hill's vortex-like internal solution are presented. 
$\lambda=0.5, \kappa=1.5$, roughly $1 / 6$ of the value of $t_{s a t}^{\prime} \approx 0.14$ for the Hill's vortex-like cases.

In Figure 20 the normalized mass (heat) flux at the drop surface is shown for four cases at a timestep after saturation is reached. The mass (heat) flux is in this case normalized by its maximum value at the drop surface at the chosen timestep. In Figure 20 (a) and (b) a case with a Hill's vortex-like internal solution is shown, whereas in (c) and (d) a case with two symmetry planes is presented. At low Peclet numbers ((a) and (c)), the highest mass flux is found in the front part of the sphere. In the case with the alternative mode (with two symmetry planes) the maximum mass flux is more concentrated in the front and the contour lines are not cylindrical symmetric, due to the influence of the non-cylindrical symmetric flow field. At higher Peclet numbers (here cases at $P e=1200$ are shown exemplary) the mass flux distribution for the Hill's vortex-like cases and cases with an alternative mode differ more severely. In the former case, the highest mass fluxes now occur on the sides of the drop, where the streamlines are closest (see Figure 5), which means that diffusion is limiting. In the latter, a band of high mass flux is obtained in the front which is aligned with the streamlines as visualized in Figure 7. This means that fluid from zones with low concentration in the inner of the drop is transported to the front and diffusion is no longer the limiting factor.

It was shown that the transfer inside liquid spheres is substantially influenced by the mode of internal circulation. In the parameter range studied, the asymptotic Sherwood number for cases with an alternative internal flow mode seems to reach no limiting value for $P e_{i} \rightarrow \infty$, which is in strong contrast to the cylindrical symmetric (Hill's vortex-like) cases, where $S h_{\infty} \approx 20$ for $P e_{i} \rightarrow \infty$. This observation is in accordance with the findings from the study of the flow structures inside the drop (see for example the discussion of the case at $R e=150$, 


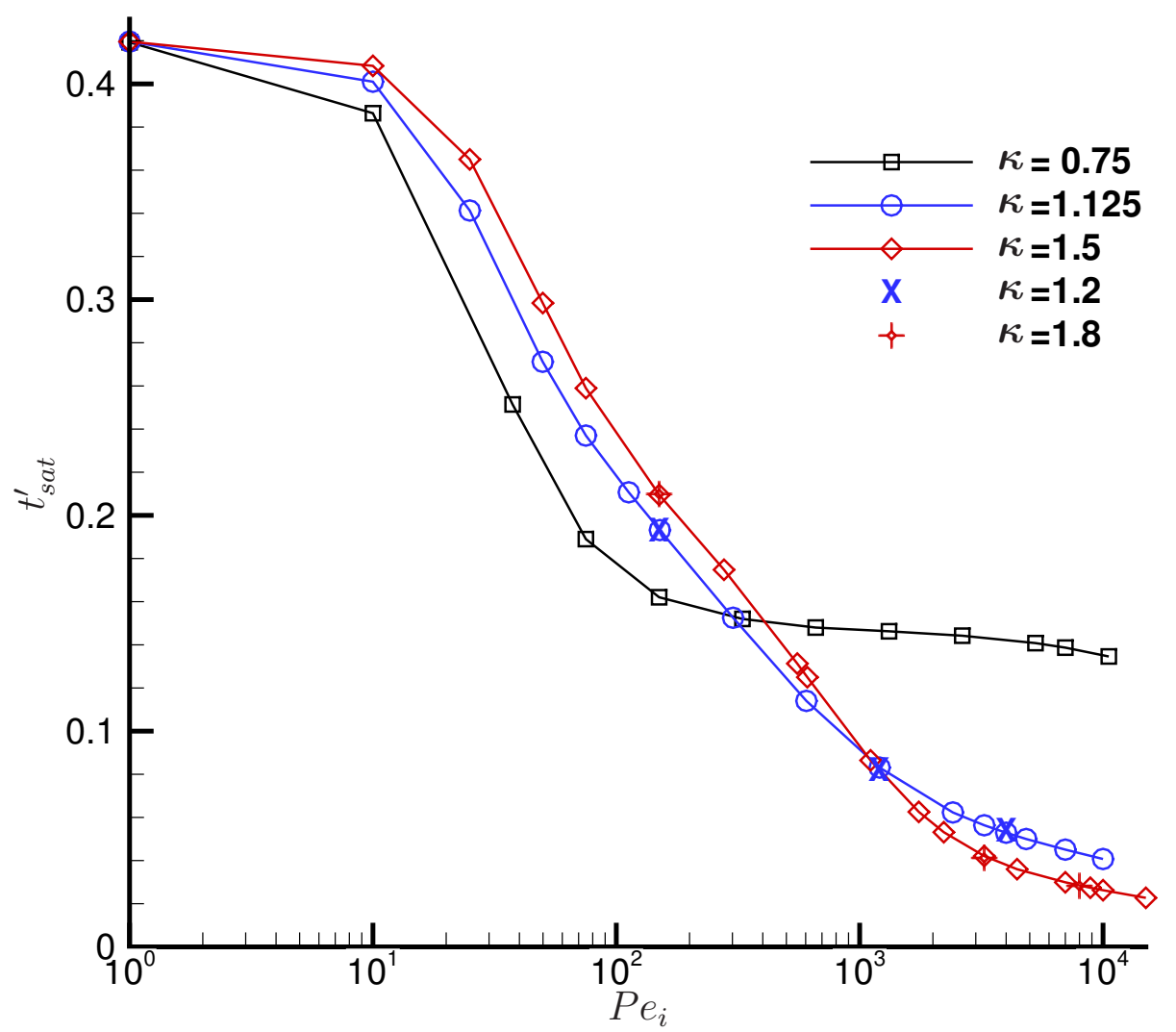

Figure 18: Nondimensional saturation time versus Peclet number at $R e=200$ and $\lambda=1.5$ with varying $\kappa$ (liquid-liquid regime).

$\lambda=0.5, \kappa=1.5$ in section 5.1). It was stated that a single streamline connects all fluid particle in a hemisphere (cases with one plane of symmetry) or quarter-sphere (cases with two planes of symmetry). Thus, a particle directly adjacent to the surface transports heat/mass to every point in a hemisphere (quarter-sphere) via convection. This means that in these cases, at high Peclet numbers diffusion is no longer the limiting factor, which is in contrast to the Hill's vortex-like cases where diffusion is limiting because of the closed twodimensional streamlines. 


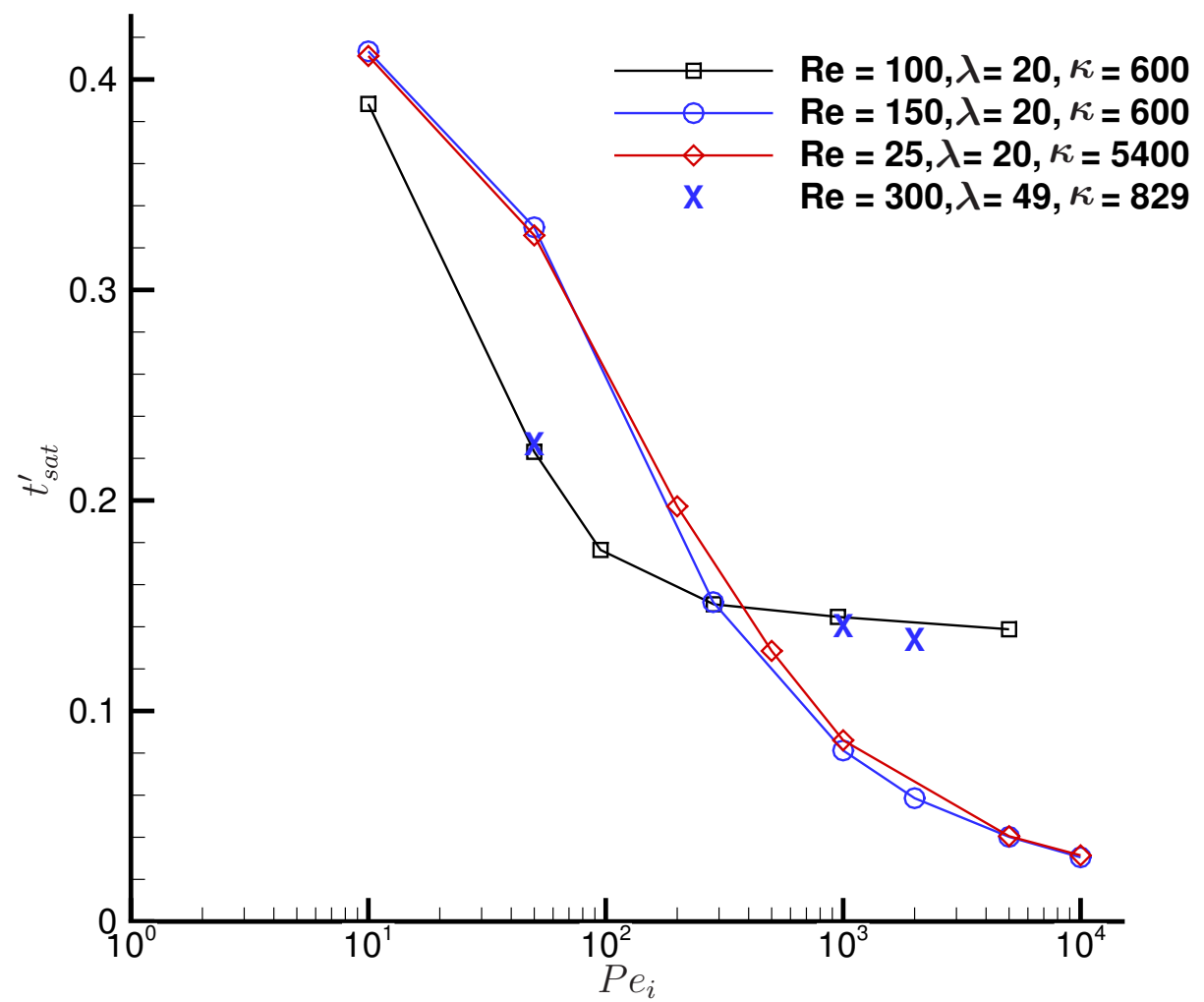

Figure 19: Nondimensional saturation time versus Peclet number in the liquid-gas regime. At $R e=100, \lambda=20, \kappa=600$, results for a case with a Hill's vortex-like internal solution are presented. 
(a)
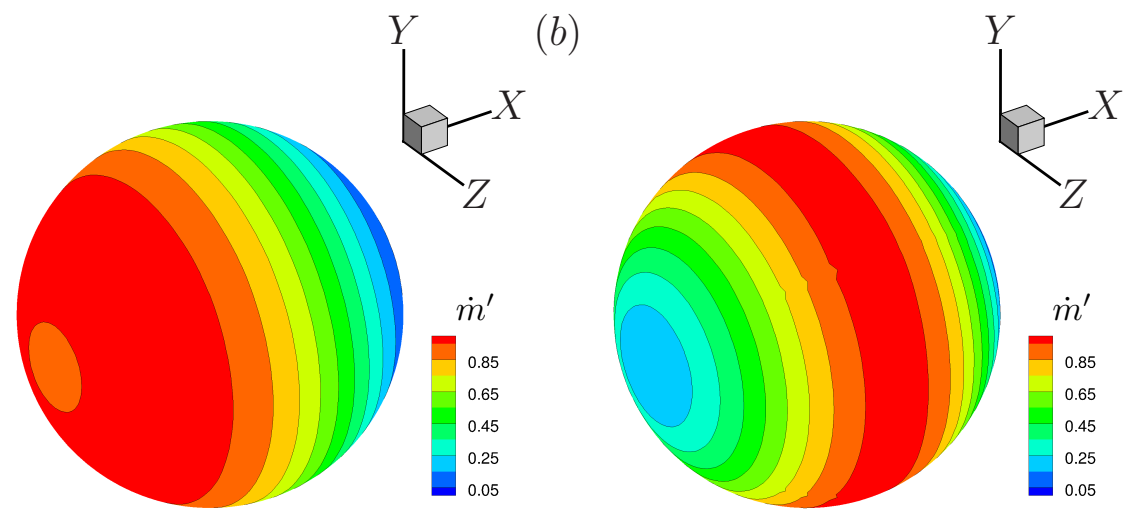

(c)
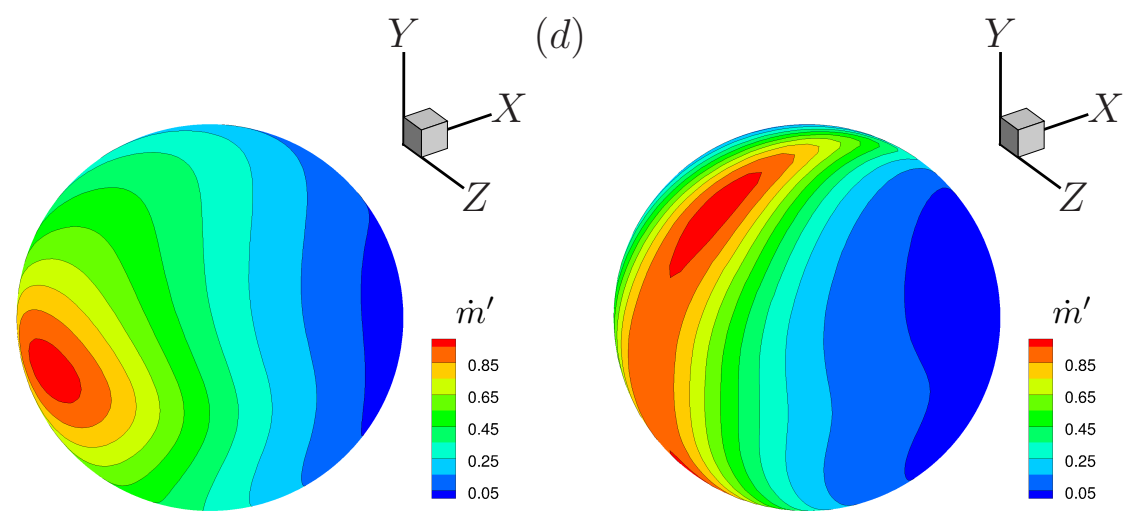

Figure 20: Normalized mass (heat) flux at the drop surface after saturation is reached.

(a) $R e=200, \lambda=0.5, \kappa=0.75$ at $P e_{i}=75(P e=68)$

(b) $R e=200, \lambda=0.5, \kappa=0.75$ at $P e_{i}=1320(P e=1200)$

(c) $R e=200, \lambda=0.5, \kappa=1.5$ at $P e_{i}=75(P e=81)$

(d) $R e=200, \lambda=0.5, \kappa=1.5$ at $P e_{i}=1109(P e=1200)$ 


\section{Conclusions}

In the present work, the results of detailed, three-dimensional numerical simulations of fixed spherical drops in a uniform flow were presented. The fluid dynamics inside and outside the drops as well as the internal problem of heat/mass transfer were discussed. Both drops in a liquid (liquid-liquid system) and a gaseous ambient phase (liquid-gas system) were considered. Special emphasis was put on cases where the inner flow field differs greatly from the Hill's vortex solution and other modes of internal circulation are observed.

The inner Reynolds number $\left(R e_{i}=\left(U_{i} d \rho_{i}\right) / \mu_{i}\right)$ seems to be the governing parameter for the appearance of alternative modes. At low Reynolds numbers, the inner flow field resembles a Hill's vortex. However, at higher Reynolds numbers the cylindrical symmetry of the inner flow field is broken. Three stable alternative types of internal modes were found. A steady solution with one symmetry plane and a steady solution with two symmetry planes and a quasi-steady, twofold rotational symmetric solution.

In the liquid-liquid regime, the inner flow field strongly alters the flow field around the drop and the flow partly detaches from the drop. This leads to a severe increase in pressure drag and a moderate increase in friction drag. In the liquid-gas regime, the outer flow field is only minimally influenced by the inner flow field, as the high viscosity ratio leads to very low velocities at the drop surface.

The finding of an exact criterion for the occurrence of alternative modes seems difficult. In the liquid-liquid regime, a threshold value of $R e_{i} \approx 400$ was found. However, this should be carefully checked as only a very limited range of parameters was studied. In the liquid-gas regime, two solutions for the same set of parameters were discovered. Depending on the initial conditions, alternative modes were observed at internal Reynolds numbers below 200 and Hill's vortex- 
like solutions at internal Reynolds numbers above 300. Even though no such bifurcation (with two stable solutions) was found in the liquid-liquid regime, it presumably exists for suitable parameters.

The internal problem of heat/mass transfer is strongly influenced by the mode of internal circulation. In the Hill's vortex-like cases, the streamlines are twodimensional and closed, with an ellipsoidal shape. This means that at high Peclet numbers the diffusion is the limiting factor, leading to asymptotic Sherwood numbers in the limit $t \rightarrow \infty, P e \rightarrow \infty$ of approximately 20. For the alternative modes, complex three-dimensional streamlines are obtained, connecting the inner fluid particles. It follows that diffusion is theoretically no longer the limiting factor and thus $S h_{\infty}=\infty$ for $t \rightarrow \infty, P e \rightarrow \infty$. Up to $P e_{i}=15,000$ this theory was confirmed and asymptotic Sherwood numbers of up to $\approx 130$ were obtained, exceeding the value of the Hill's vortex-like solutions by more than a factor of six. However, for moderate Peclet numbers up to $P e_{i}=300$, it was found that the heat/mass transfer is faster in the Hill's vortex-like cases. From the present results, it seems that in many cases full three-dimensional simulations are necessary to capture all features of the flow field outside and inside of drops. The assumption of axisymmetry may lead to wrong results concerning for example the drag and the heat/mass transfer. Especially in liquid-liquid systems, the alternative modes studied in the present paper should exist in many technical important cases, as long as the spherical droplet shape is retained. In the liquid-gas regime physically meaningful cases seem possible. However, the influence of evaporation needs to be quantified.

\section{Appendix A. Drag coefficient correlations}

In the following, the drag coefficient correlations used in Figure 9 and defined by Feng and Michaelides (2001) are given. For a viscous sphere with $0 \leq \lambda \leq 2$ 
and $5<R e \leq 1000$ :

$$
C_{D}(R e, \lambda)=\frac{2-\lambda}{2} C_{D}(R e, 0)+\frac{4 \lambda}{6+\lambda} C_{D}(R e, 2),
$$

where the functions for $C_{D}(R e, 0)$ and $C_{D}(R e, 2)$ are given by:

$$
\begin{gathered}
C_{D}(R e, 0)=\frac{48}{R e}\left(1+\frac{2.21}{\sqrt{R e}}-\frac{2.14}{R e}\right) \\
C_{D}(R e, 2)=17.0 R e^{-2 / 3}
\end{gathered}
$$

For rigid spheres the correlation by Putnam (1961) is used:

$$
C_{D}(R e, \infty)=\frac{24}{R e}\left(1+\frac{1}{6} R e^{2 / 3}\right)
$$


Table 7: Drag coefficients for viscous spheres in the liquid-gas regime. Some simulations were started from a converged previous simulation. The number in brackets indicates the original value from the previous simulation.

\begin{tabular}{cccccccc}
\hline \multirow{2}{*}{$R e$} & \multirow{2}{*}{$\kappa$} & \multirow{2}{*}{$R_{i}$} & \multicolumn{2}{c}{$C_{D}$} & $C_{D, f}$ & \multirow{2}{*}{$C_{D, p}$} \\
& & & \multicolumn{2}{c}{ Mesh 1 } & Mesh 2 & & \\
\hline 25 & 20 & 3,600 & 259 & 2.306 & - & 1.422 & 0.884 \\
25 & 20 & 4,800 & 343 & 2.305 & - & 1.422 & 0.883 \\
50 & 20 & $120(2,400)$ & 25 & 1.535 & - & 0.889 & 0.646 \\
50 & 20 & 1,200 & 223 & 1.528 & - & 0.890 & 0.638 \\
50 & 20 & 1,800 & 329 & 1.527 & - & 0.890 & 0.637 \\
$50(150)$ & 20 & 600 & 116 & 1.530 & - & 0.888 & 0.642 \\
100 & 20 & 600 & 285 & 1.046 & - & 0.563 & 0.483 \\
\hline 300 & 49 & 829 & 303 & 0.632 & 0.631 & 0.274 & 0.358 \\
\hline 25 & 20 & 5,400 & 227 & 2.346 & - & 1.446 & 0.900 \\
\hline 25 & 20 & 6,000 & 243 & 2.347 & - & 1.446 & 0.901 \\
25 & 20 & 7,200 & 360 & 2.350 & - & 1.448 & 0.902 \\
50 & 20 & $1,200(2,400)$ & 170 & 1.558 & - & 0.903 & 0.655 \\
50 & 20 & $1,800(2,400)$ & 212 & 1.551 & - & 0.900 & 0.651 \\
50 & 20 & 2,400 & 250 & 1.561 & - & 0.905 & 0.656 \\
$100(150)$ & 20 & 600 & 201 & 1.071 & - & 0.568 & 0.503 \\
130 & 20 & 600 & 250 & 0.941 & - & 0.464 & 0.477 \\
150 & 20 & 600 & 284 & 0.877 & 0.876 & 0.434 & 0.443 \\
\hline
\end{tabular}


Table 8: Asymptotic Sherwood numbers for viscous spheres on different meshes. The ${ }^{+}$ indicates that the asymptotic value is not steady but oscillating.

\begin{tabular}{cccccccc}
\hline$R e$ & $\lambda$ & $\kappa$ & $P e$ & $P e_{i}$ & & $\begin{array}{c}S h_{\infty} \\
\text { Mesh 2 }\end{array}$ & Mesh 3 \\
\hline 200 & 0.5 & 0.75 & 300 & 330 & 18.61 & 18.57 & - \\
200 & 0.5 & 0.75 & 4,800 & 5279 & 19.71 & 19.1 & - \\
200 & 0.5 & 0.75 & 9,600 & 10559 & 20.68 & 19.44 & - \\
\hline 300 & 49 & 829 & 16,734 & 1,000 & 19.97 & 19.77 & - \\
\hline 200 & 0.5 & 1.125 & 150 & 151 & 15.64 & 15.59 & - \\
200 & 0.5 & 1.125 & 9,931 & 10000 & 72.18 & 70.39 & - \\
\hline 200 & 0.5 & 1.5 & 163 & 150 & 13.99 & 13.92 & - \\
200 & 0.5 & 1.5 & 2,400 & 2,217 & 63.97 & - & 63.7 \\
200 & 0.5 & 1.5 & 9,600 & 8,868 & 113.37 & - & 112.43 \\
200 & 0.5 & 1.5 & 10,824 & 10,000 & 117.81 & 116.75 & - \\
\hline 150 & 20 & 600 & 15,873 & 1,000 & $39.39^{+}$ & $39.92^{+}$ & - \\
150 & 20 & 600 & 31,746 & 2,000 & $54.31^{+}$ & $54.77^{+}$ & - \\
150 & 20 & 600 & 158,729 & 10,000 & $103^{+}$ & $102.5^{+}$ & - \\
\hline
\end{tabular}


Abdel-Alim, A. H., Hamielec, A. E., 1975. A theoretical and experimental investigation of the effect of internal circulation on the drag of spherical droplets falling at terminal velocity in liquid media. Industrial \& Engineering Chemistry Fundamentals 14 (4), 308-312.

Abramzon, B., Sirignano, W., 1989. Droplet vaporization model for spray combustion calculations. International Journal of Heat and Mass Transfer 32 (9), $1605-1618$.

Bagchi, P., Balachandar, S., Sep 2002. Steady planar straining flow past a rigid sphere at moderate Reynolds number. J. Fluid Mech. 466, 365-407.

Boyde, J. M., Le Clercq, P. C., Di Domenico, M., Aigner, M., 2013. Extension of the turbulent flame speed closure model to ignition in multiphase flows. Combustion and Flame 160 (2), 351-365.

Clift, R., Grace, J., Weber, M., 1978. Bubbles, drops, and particles. Academic Press, New York.

Colombet, D., Legendre, D., Cockx, A., Guiraud, P., 2013. Mass or heat transfer inside a spherical gas bubble at low to moderate Reynolds number. International Journal of Heat and Mass Transfer 67, 1096 - 1105.

Domenico, M. D., Gerlinger, P., Aigner, M., 2010. Development and validation of a new soot formation model for gas turbine combustor simulations. Combustion and Flame 157 (2), $246-258$.

Eckel, G., Rachner, M., Le Clercq, P., Aigner, M., 2016. Semi-empirical model for the unsteady shear breakup of liquid jets in cross-flow. Atomization and Sprays $26(7), 687-712$.

Engberg, R. F., Kenig, E. Y., 2015. An investigation of the influence of initial 
deformation on fluid dynamics of toluene droplets in water. International Journal of Multiphase Flow 76, $144-157$.

Feng, Z.-G., Michaelides, E. E., 2001. Drag coefficients of viscous spheres at intermediate and high Reynolds numbers. Journal of Fluids Engineering 123 (4), 841-849.

Ghidersa, B., Dusek, J., 11 2000. Breaking of axisymmetry and onset of unsteadiness in the wake of a sphere. Journal of Fluid Mechanics 423, 33-69.

Handlos, A. E., Baron, T., 1957. Mass and heat transfer from drops in liquidliquid extraction. AIChE Journal 3 (1), 127-136.

Hill, M. J. M., 1894. On a spherical vortex. Philosophical Transactions of the Royal Society of London A: Mathematical, Physical and Engineering Sciences $185,213-245$.

Ivanova, E., Di Domenico, M., Noll, B., Aigner, M., 2009. Unsteady Simulations of Flow Field and Scalar Mixing in Transverse Jets. ASME paper GT200959147.

Jin, J., Borman, G., 1985. A model for multicomponent droplet vaporization at high ambient pressures. SAE Technical Paper 850264.

Johnson, T. A., Patel, V. C., 1 1999. Flow past a sphere up to a Reynolds number of 300. Journal of Fluid Mechanics 378, 19-70.

Juncu, G., 2010. A numerical study of the unsteady heat/mass transfer inside a circulating sphere. International Journal of Heat and Mass Transfer 53 (1516), $3006-3012$.

Kim, D., Choi, H., 6 2002. Laminar flow past a sphere rotating in the streamwise direction. Journal of Fluid Mechanics 461, 365-386. 
Knopp, T., Zhang, X., Kessler, R., Lube, G., 2010. Enhancement of an industrial finite-volume code for large-eddy-type simulation of incompressible high Reynolds number flow using near-wall modelling. Computer Methods in Applied Mechanics and Engineering 199 (1316), 890 - 902.

Kronig, R., Brink, J. C., 1951. On the theory of extraction from falling droplets. Applied Scientific Research 2 (1), 142-154.

LeClair, B. P., Hamielec, A. E., Pruppacher, H. R., Hall, W. D., 1972. A theoretical and experimental study of the internal circulation in water drops falling at terminal velocity in air. Journal of the Atmospheric Sciences 29 (4), $728-740$.

Löwe, J., Probst, A., Knopp, T., Kessler, R., jan 2015. A low-dissipation lowdispersion second-order scheme for unstructured finite-volume flow solvers. In: AIAA SciTech. American Institute of Aeronautics and Astronautics.

Newman, A. B., 1931. The drying of porous solids: Diffusion and surface emission equations. Transactions of the American Institute of Chemical Engineers 27 (1), 203-220.

Oliver, D. L. R., Chung, J. N., 4 1987. Flow about a fluid sphere at low to moderate Reynolds numbers. Journal of Fluid Mechanics 177, 1-18.

Paschedag, A., Piarah, W., Kraume, M., 2005. Sensitivity study for the mass transfer at a single droplet. International Journal of Heat and Mass Transfer 48 (16), $3402-3410$.

Putnam, A., 1961. Integratable form of droplet drag coefficient. Ars Journal 31 (10), 1467-1468.

Rivkind, V. Y., Ryskin, G. M., 1976. Flow structure in motion of a spherical 
drop in a fluid medium at intermediate Reynolds numbers. Fluid Dynamics $11(1), 5-12$.

Sugioka, K.-I., Komori, S., 1 2007. Drag and lift forces acting on a spherical water droplet in homogeneous linear shear air flow. Journal of Fluid Mechanics $570,155-175$.

Talley, D., Yao, S., 1988. A semi-empirical approach to thermal and composition transients inside vaporizing fuel droplets. Symposium (International) on Combustion 21 (1), $609-616$.

Thorsen, G., Stordalen, R., Terjesen, S., 1968. On the terminal velocity of circulating and oscillating liquid drops. Chemical Engineering Science 23 (5), $413-426$.

Waheed, M. A., 2001. Fluiddynamik und Stoffaustausch bei freier und erzwungener Konvektion umströmter Tropfen. Ph.D. thesis, Technische Hochschule Aachen.

Wegener, M., Kraume, M., Paschedag, A. R., 2010. Terminal and transient drop rise velocity of single toluene droplets in water. AIChE Journal 56 (1), 2-10.

Wegener, M., Paul, N., Kraume, M., 2014. Fluid dynamics and mass transfer at single droplets in liquid/liquid systems. International Journal of Heat and Mass Transfer 71, $475-495$. 